\title{
Magnetic Braking of Sun-like and Low-mass Stars: Dependence on Coronal Temperature
}

\author{
George Pantolmos (i] and Sean P. Matt (10 \\ University of Exeter, Department of Physics \& Astronomy, Physics Building, Stocker Road, Exeter, EX4 4QL, UK; gp323@exeter.ac.uk \\ Received 2017 June 23; revised 2017 September 20; accepted 2017 September 28; published 2017 November 6
}

\begin{abstract}
Sun-like and low-mass stars possess high-temperature coronae and lose mass in the form of stellar winds, which are driven by thermal pressure and complex magnetohydrodynamic processes. These magnetized outflows probably do not significantly affect the star's structural evolution on the main sequence, but they brake the stellar rotation by removing angular momentum, a mechanism known as magnetic braking. Previous studies have shown how the braking torque depends on the magnetic field strength and geometry, stellar mass and radius, mass-loss rate, and rotation rate of the star, assuming a fixed coronal temperature. For this study, we explore how different coronal temperatures can influence the stellar torque. We employ 2.5D, axisymmetric, magnetohydrodynamic simulations, computed with the PLUTO code, to obtain steady-state wind solutions from rotating stars with dipolar magnetic fields. Our parameter study includes 30 simulations with different coronal temperatures and surface magnetic field strengths. We consider a Parker-like (i.e., thermal-pressure-driven) wind, and therefore coronal temperature is the key parameter determining the velocity and acceleration profile of the flow. Since the mass-loss rates for these types of stars are not well-constrained, we determine how the torque scales for a vast range of stellar mass-loss rates. Hotter winds lead to faster acceleration, and we show that (for a given magnetic field strength and mass-loss rate) a hotter outflow leads to a weaker torque on the star. We derive new predictive torque formulae that quantify this effect over a range of possible wind acceleration profiles.
\end{abstract}

Key words: magnetohydrodynamics (MHD) - stars: low-mass - stars: magnetic field - stars: rotation - stars: solartype - stars: winds - outflows

\section{Introduction}

Stellar winds are very common phenomena in our universe. For Sun-like and low-mass stars $\left(M_{*} \lesssim 1.3 M_{\odot}\right)$, such outflows are usually in the form of coronal winds (Parker 1958, 1963), due to their origin in the several MK stellar hot coronae. Although the effect of coronal winds on stellar mass during a star's main-sequence (MS) life is relatively small, they can influence the environment of surrounding planets (e.g., Lüftinger et al. 2015) and have an enormous impact on stellar rotation by exerting a spin-down torque on the stellar surface (e.g., Schatzman 1962; Weber \& Davis 1967). Hence, over the years, the angular-momentum (or rotational) evolution of cool stars has been the subject of very intensive studies (for a review, see Bouvier et al. 2014).

The spin down of MS cool stars was established observationally by early studies (Kraft 1967; Skumanich 1972) that showed the rotation periods of these types of stars increasing as the stellar age advances. The current picture of the rotational evolution of cool stars is more complicated, and observations (e.g., Barnes 2003, 2010; Irwin \& Bouvier 2009; Meibom et al. $2011,2015)$ show that stellar rotation depends on both mass and age. In addition, the observed trends between magnetic activity (or coronal X-ray emission) and stellar rotation (e.g., Pizzolato et al. 2003; Wright et al. 2011), and the observed evolution of stellar magnetic properties (e.g., Vidotto et al. 2014a; See et al. 2015) suggest that solar- and late-type stars lose mass and angular momentum in the form of magnetized outflows.

Coronal-wind modeling, using analytic theory (e.g., Parker 1958; Weber \& Davis 1967; Mestel 1968; Heinemann \& Olbert 1978; Low \& Tsinganos 1986) or iterative methods/ numerical simulations (e.g., Pneuman \& Kopp 1971;
Sakurai 1985; Washimi \& Shibata 1993; Keppens \& Goedbloed 2000; Cohen et al. 2007; Vidotto et al. 2009), has a long history in the literature. The main source for understanding the nature, properties, and dynamics of coronal winds comes from direct observations of the solar wind. The solar corona expands into interplanetary space in the form of a supersonic, magnetized wind that evolves during a solar cycle. Near the solar minimum, the solar wind is bimodal with a fast, tenuous, and steady component emanating from large polar coronal holes and a slower, denser, and filamentary component emerging from the top of the helmet streamers originating from the magnetic activity belt (e.g., McComas et al. 2007, 2008). During the solar maximum, the solar wind becomes more variable and is more dominated by the slow wind at all latitudes (e.g., McComas et al. 2003, 2007). The solar wind is a direct consequence of the hot solar corona (with $T>10^{6} \mathrm{~K}$ ), and thus the solar plasma acceleration (for both the fast and slow solar winds) is connected to the coronal heating problem (e.g., De Moortel \& Browning 2015). The physical mechanisms responsible for the solar coronal heating are still being debated, but they all require magnetic fields as a key ingredient (see, e.g., Aschwanden 2005; Klimchuk 2015; Velli et al. 2015). The solar magnetic field (a product of the solar dynamo that operates within the convection zone) threads the solar photosphere, expands throughout the solar atmosphere, and eventually connects with and energizes the solar wind. Recent advances in solar-wind theory include wave dissipation (via turbulence) and magnetic reconnection as heat sources for the expanding outer solar atmosphere (see, e.g., Ofman 2010; Cranmer 2012; Hansteen \& Velli 2012; Cranmer et al. 2015). Scaling-law models (e.g., Wang \& Sheeley 1991; Fisk 2003; Schwadron \& McComas 2003, 2008) reproduce part of the 
observed characteristics of the solar wind, although that approach does not treat the coronal heating/solar-wind acceleration problem in a self-consistent way (see, e.g., Hansteen \& Velli 2012). A conclusive answer on what heats the solar corona and what physical processes drive the solar wind does not exist. X-ray observations have revealed the existence of hot outer atmospheres in every low-mass star (e.g., Wright et al. 2011). However, it is still not clear how coronal heating should vary among late-type stars with varying masses and rotation rates, and what this indicates for the observed trends in X-ray emission (see, e.g., Testa et al. 2015). Therefore, it is still an open question of how to apply our knowledge of the solar coronal heating and wind acceleration to other stars. The present work is concerned with characterizing the global torques on stars and how they scale for a variety of stellar properties, while solutions to the coronal heating problem remain uncertain. Consequently, in this work, we treat many of the coronal processes as "free parameters," including the wind mass-loss rates and wind acceleration profiles, which show how the uncertainties in our understanding of stellar coronae will influence our ability to predict angular-momentum loss.

Early works (e.g., Schatzman 1962; Mestel 1984; Mestel \& Spruit 1987; Kawaler 1988) have provided analytic prescriptions for the magnetic braking of cool stars in the framework of stellar-torque theory, and some more recent works compute the stellar angular-momentum losses self-consistently via multidimensional numerical simulations. For example, studies have quantified how the magnetic braking scales with various stellar parameters (e.g., Matt \& Pudritz 2008; Matt et al. 2012; Cohen $\&$ Drake 2014), and others have shown how stellar angularmomentum losses depend on different magnetic field geometries (e.g., Garraffo et al. 2015, 2016; Réville et al. 2015a; Finley \& Matt 2017). With the new advances in ZeemanDoppler Imaging (e.g., Donati \& Brown 1997; Donati \& Landstreet 2009), observers can now extract stellar-surface magnetic field maps that can be used to reconstruct the stellar field near the star. Some studies (e.g., Vidotto et al. 2014b; Alvarado-Gómez et al. 2016; Réville et al. 2016a) have used such maps in their wind simulations in order to provide trends for stellar torques based on realistic magnetic fields. In general, accurate stellar-torque predictions are one of the critical ingredients for rotational evolution models (e.g., Reiners \& Mohanty 2012; Gallet \& Bouvier 2013, 2015; Johnstone et al. 2015a; Matt et al. 2015; Amard et al. 2016; See et al. 2017b).

Coronal temperatures among MS cool stars significantly vary (e.g., Johnstone et al. 2015b). However, there has not yet been a systematic study of magnetic braking that investigates the key parameters (i.e., stellar coronal temperature and polytropic index) that affect the wind driving (or flow acceleration and velocity). The objective of this study is to quantify the influence of different flow temperatures on stellar torques. We adopt the approach introduced in Matt \& Pudritz (2008). In particular, Matt \& Pudritz (2008) found that the effective magnetic lever arm (or Alfvén radius), which determines the efficiency of the braking torque, is a power law in the parameter $\Upsilon$ (i.e., wind magnetization), which depends on the stellar mass, radius, mass-loss rate, and magnetic field strength. Studies on massive, hot stars (e.g., type-O stars; see Ud-Doula et al. 2009) have found similar scalings between the stellar parameters and angular-momentum losses, with the main difference being that the wind-driving mechanism is fundamentally different (e.g., Lamers \& Cassinelli 1999; Owocki et al. 2009). Following Matt \& Pudritz (2008), a series of studies showed how to include the dependence of the braking torque on the stellar spin rate (Matt et al. 2012) and the different magnetic field geometries (Réville et al. 2015a, 2016a; Finley \& Matt 2017). All of these studies (Matt \& Pudritz 2008; Matt et al. 2012; Réville et al. 2015a, 2016a; Finley \& Matt 2017) used polytropic Parker wind models (e.g., Parker 1963; Keppens \& Goedbloed 1999; Lamers \& Cassinelli 1999) modified by rotation and magnetic fields. However, they kept fixed the flow thermodynamics parameters (i.e., coronal temperature and polytropic index) that determine the wind velocity and acceleration.

The purpose of this paper is to examine and quantify how variations in the coronal temperature (one of the key parameters that influence the wind acceleration) will affect the stellar angular-momentum loss by employing $2.5 \mathrm{D}$, ideal $\mathrm{MHD}$, and axisymmetric simulations. In the following section (Section 2), we provide a brief theoretical discussion of the concept of angular-momentum loss due to stellar outflows. In Section 3, we discuss how our numerical setup is suitable for studying a wide range of wind acceleration profiles and describe our parameter space. In Section 4, we focus on the results of this study, and we show the braking laws for different temperatures. In Section 5, two new torque formulae that are independent of the flow temperature are proposed, and finally in Section 6, the main conclusions of this paper are summarized. In Appendices A and B, we discuss some numerical issues in our simulations. Appendix $\mathrm{C}$ provides an empirical approach to predict stellar torques for any temperature. Finally, Appendix D contains plots of the complete simulation grid for this parameter study.

\section{Magnetized Outflows and Efficiency of Angular- Momentum Loss}

In general, the total angular-momentum rate carried away from a star in a stellar wind can be written as

$$
\tau_{w}=\dot{M}_{w} \Omega_{*}\left\langle R_{\mathrm{A}}\right\rangle^{2},
$$

where $\dot{M}_{w}$ is the integrated stellar mass-loss rate due to the wind, $\Omega_{*}$ is the stellar rotation rate, and $\left\langle R_{\mathrm{A}}\right\rangle^{2}$ is the square of a characteristic length scale in the wind. Using a mechanical analogy, $\left\langle R_{\mathrm{A}}\right\rangle$ can be thought of as a "lever arm length" that determines the efficiency of the torque on the star exerted by the plasma efflux. Generically, this efficiency of the angularmomentum loss can be expressed as the ratio of this lever arm length to the stellar radius, $R_{*}$,

$$
\frac{\left\langle R_{\mathrm{A}}\right\rangle}{R_{*}} \equiv\left(\frac{\tau_{w}}{\dot{M}_{w} \Omega_{*} R_{*}^{2}}\right)^{1 / 2} .
$$

The precise value for the length scale $\left\langle R_{\mathrm{A}}\right\rangle$ depends on the detailed (and multidimensional) physics of the wind. As an example, a spherically symmetric, inviscid, hydrodynamical wind would simply carry away the specific angular momentum it has from the stellar surface. Thus, the star is subjected to an angular-momentum loss that gives $\left\langle R_{\mathrm{A}}\right\rangle / R_{*}=(2 / 3)^{1 / 2}$ (e.g., Mestel 1968), which deviates from unity because the torque 
depends on the distance from the rotation axis (i.e., cylindrical $\varpi=r \sin \theta$ ), not on the spherical radius $r$.

In a magnetized wind, Lorentz forces transmit angular momentum from the star to the wind, even after it has left the stellar surface, which can significantly increase the efficiency of angular-momentum loss. Weber \& Davis (1967; see also Schatzman 1962) showed that for a one-dimensional, magnetized flow along the stellar equator, under the assumption of steady-state, ideal MHD, this radius equals the radial Alfvén radius, defined as the radial distance where the wind speed equals the local Alfvén speed (considering only the radial components of the velocity and magnetic field). In a two- or three-dimensional ideal MHD flow, the value of $\left\langle R_{\mathrm{A}}\right\rangle^{2}$ is the mass-loss-weighted average of the square of the poloidal Alfvén (cylindrical) radius (Washimi \& Shibata 1993).

In our simulations, $\Omega_{*}$ and $R_{*}$ are specified as input parameters, and we directly compute the resulting values of $\tau_{w}$ and $\dot{M}_{w}$ in the wind solutions (see below). Thus, following Matt \& Pudritz (2008; and Matt et al. 2012; Réville et al. 2015a, 2016a; Finley \& Matt 2017), we compute the value of $\left\langle R_{\mathrm{A}}\right\rangle / R_{*}$ using Equation (2) and refer to this throughout as the "torque-averaged Alfvén radius" or "effective Alfvén radius." Note that defining $R_{A}$ in this way does not depend on any assumptions about the physics of the angular-momentum transfer (e.g., it does not require a steady state, nor assume ideal MHD conditions); the value $\left(\left\langle R_{\mathrm{A}}\right\rangle / R_{*}\right)^{2}$ simply represents a dimensionless torque. Also, the scaling laws we derive below for predicting $\left\langle R_{\mathrm{A}}\right\rangle$ are, by definition, the appropriate length scale to use in Equation (1) for computing the global torque.

\section{Stellar Wind Solutions}

\subsection{Numerical Setup}

This study employs ideal MHD and axisymmetric simulations, using the PLUTO code (Mignone et al. 2007) in a 2.5D computational grid (i.e., two spatial coordinates with three vector components), in order to obtain steady-state (or quasisteady-state) stellar wind solutions. PLUTO numerically solves the following set of ideal MHD conservation laws:

$$
\begin{gathered}
\partial_{t} \rho+\nabla \cdot \rho \boldsymbol{v}=0, \\
\partial_{t} \boldsymbol{m}+\nabla \cdot\left(\boldsymbol{m} \boldsymbol{v}-\boldsymbol{B B}+\boldsymbol{I} p_{\mathrm{tot}}\right)=\rho \boldsymbol{g}, \\
\partial_{t} E+\nabla \cdot\left[\left(E+p_{\mathrm{tot}}\right) \boldsymbol{v}-\boldsymbol{B}(\boldsymbol{v} \cdot \boldsymbol{B})\right]=\boldsymbol{m} \cdot \boldsymbol{g}, \\
\partial_{t} \boldsymbol{B}+\nabla \cdot(\boldsymbol{v} \boldsymbol{B}-\boldsymbol{B} \boldsymbol{v})=0,
\end{gathered}
$$

where $\partial_{t} \equiv \partial / \partial t$ denotes the time derivative operator and $\boldsymbol{I}$ is the identity matrix. The mass density is denoted by $\rho, p_{\text {tot }}=p+\boldsymbol{B}^{2} / 2$ is the total pressure, composed of the thermal pressure, $p$, and the magnetic pressure, ${ }^{1} \boldsymbol{B}^{2} / 2$. The velocity field is $\boldsymbol{v}, \boldsymbol{m}=\rho \boldsymbol{v}$ is the momentum density, $\boldsymbol{B}$ is the magnetic field, and $\boldsymbol{g}=-\left(G M_{*} / r^{2}\right) \hat{r}$ represents the gravitational acceleration, where $G$ is Newton's gravitational constant, $M_{*}$ is the stellar mass, $r$ is the distance to center of the star, and $\hat{r}$ stands for the radial unit vector. The total energy density is $E=\rho \boldsymbol{e}+\boldsymbol{m}^{2} /(2 \rho)+\boldsymbol{B}^{2} / 2$, where $e$ is the specific internal energy. Finally, we adopt an equation of state for ideal gases, $\rho e=p /(\gamma-1)$, where $\gamma$ is the adiabatic exponent.

\footnotetext{
1 In the PLUTO code, the magnetic field is defined with a factor of $1 / \sqrt{4 \pi}$ included.
}

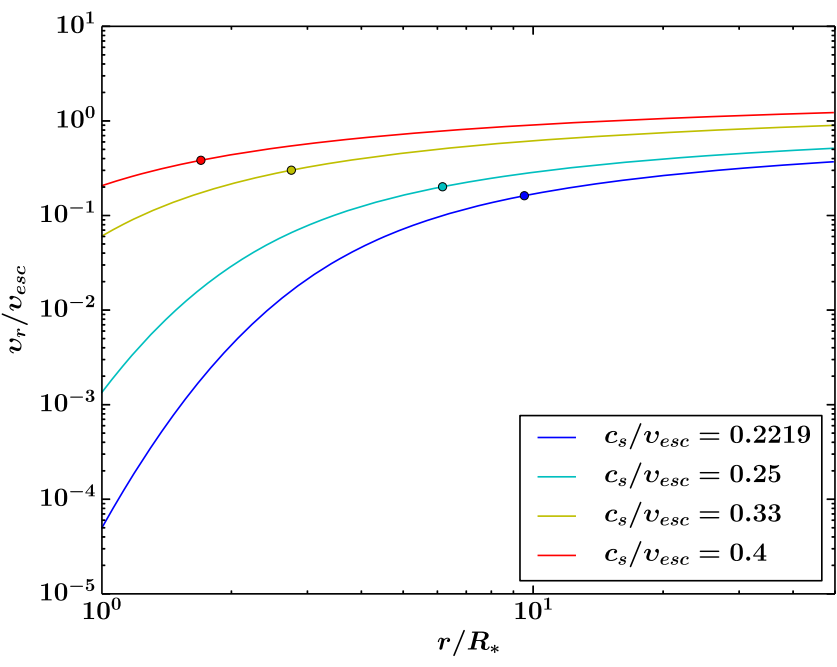

Figure 1. Flow velocity vs. radial distance for four different temperatures, here parameterized by the ratio of the adiabatic sound speed to the escape speed from the star, for $1 \mathrm{D}$ hydrodynamic winds from non-rotating stars. The above profiles are also used as the initial velocity wind profile in our simulations. The circles correspond to the radial distance at which the flow becomes supersonic. Each temperature produces a unique wind acceleration profile, and hotter winds always exhibit higher base and terminal velocities than cooler winds.

We use a second-order piecewise linear reconstruction of all primitive variables $(\rho, \boldsymbol{v}, p, \boldsymbol{B})$ with the minmod limiter the and the HLL Riemann solver (e.g., Toro 2009) to compute the fluxes in Equations (3)-(6). The induction equation (Equation (6)) is solved with the constrained transport (CT) method (Balsara \& Spicer 1999) in order to ensure that the divergence-free condition for the magnetic field will be maintained in our domain. The computational gird has spherical geometry for the spatial coordinates and covers $r \in[1,50] R_{*}$, where $R_{*}$ is the stellar radius and $\theta \in[0, \pi]$, with a total of $256 \times 512$ zones. A stretched grid is constructed along the $\hat{r}$ direction. The first grid zone at the stellar surface (i.e., the inner boundary where $r / R_{*}=1$ ) has size $\Delta r=5 \times 10^{-3} R_{*}$ but increases with $r$ such that 256 points reach $50 R_{*}$ (i.e., the outer boundary), with the last grid cell having size $\Delta r=1.015 R_{*}$. The grid is uniform along the $\hat{\theta}$ direction.

We initialize the whole computational domain with a dipole field, for which the radial and polar components are given by

$$
\begin{gathered}
B_{r}=2 B_{*}\left(\frac{R_{*}}{r}\right)^{3} \cos \theta, \\
B_{\theta}=B_{*}\left(\frac{R_{*}}{r}\right)^{3} \sin \theta,
\end{gathered}
$$

where $B_{*}$ is the equatorial surface field strength. We treat the magnetic field using the the "background field splitting" approach (Powell et al. 1999), which sets the dipole field as a time-independent component, and the code calculates the deviation from the initial field. This method provides better numerical accuracy in the treatment of the magnetic field, especially where strong gradients in the magnetic field might otherwise lead to significant numerical diffusion.

We also initialize our grid with a 1D, polytropic Parker's wind solution, shown in Figure 1, and we set the density and the thermal pressure based on the mass continuity equation and 
the polytropic relation ( $p_{\text {th }} \propto \rho^{\gamma}$ ), respectively. Further details can be found in Section 3.2.

For both boundary zones of the $\theta$ coordinate, we use an "axisymmetric" type of boundary condition, which symmetrizes all of the variables across the borders and flips the signs of the $\phi$ and normal components of the vector fields. The outer boundary condition of the $r$ coordinate is set to be "outflow," which sets the gradient of each variable to be zero across the boundary. When the code starts to evolve Equations (3)-(6) in time, the initial state is blown outwards and the steady-state solution, which we are interested in, depends only on the inner boundary conditions. Since our wind solutions only depend on the inner boundary, which represents the stellar surface, for these ghost zones we use a more sophisticated boundary condition. We keep fixed at the stellar boundary the values for the thermal pressure and density computed from the onedimensional polytropic Parker's wind we used to initialize our grid. This boundary condition corresponds to a stellar atmosphere in which its density and temperature do not vary in time and exhibit a temperature profile such that $T \propto \rho^{\gamma-1}$. Moreover, this condition ensures that the temperature of the flow does not exhibit a dependence on $\theta$ at the stellar boundary. The boundary condition for the poloidal magnetic field is forced to maintain the initial dipole state, since the flow is subAlfvénic and magnetic pressure dominates over the thermal and wind hydrodynamic pressure. For the toroidal magnetic field, we linearly extrapolate the toroidal field values calculated in the computational domain into the ghost zones. For the poloidal velocity, we also linearly extrapolate the computed value of the poloidal velocity into the ghost zones in order to have a flow velocity that increases monotonically with radius inside the ghost zones. In a steady-state and axisymmetric flow, the toroidal component of the electric field should be zero (e.g., Lovelace et al. 1986; Zanni \& Ferreira 2009), and thus we force the poloidal component of the velocity and magnetic field to be parallel to each other. The rotation is enforced only in the stellar boundary, which we accomplish by setting the boundary condition for the toroidal component of the velocity given by the equation

$$
v_{\phi}=\Omega_{*} r \sin \theta+\frac{v_{p}}{B_{p}} B_{\phi},
$$

in order to satisfy the $\boldsymbol{E}=0$ condition in a frame rotating with the star (Zanni \& Ferreira 2009). In Equation (9), $r$ is the spherical radius and the subscripts $p$ and $\phi$ stand for the poloidal and toroidal components respectively, of the velocity and magnetic field.

Each simulation is stopped when the solution converges to a steady state. Some of the obtained numerical solutions are periodic, and we discuss the steadiness, and the peculiarity of these simulations in Appendix A. We further examine the correctness of each wind solution by checking how well the five constant of motion are conserved along the flow streamlines (e.g., Keppens \& Goedbloed 2000). The numerical accuracy of our simulations is discussed in more detail in Appendix B.

\subsection{Parameters of the Study}

For purely hydrodynamic polytropic stellar winds, the two main physical parameters that determine the wind speed and acceleration are the temperature of the plasma and the
Table 1

Coronal Temperatures of the Wind Models Studied for Different Stellar Properties

\begin{tabular}{lcccc}
\hline \hline & $\begin{array}{c}\text { Temperature } \\
\left(\mathrm{MK}^{\circ}\right)\end{array}$ & $\begin{array}{c}\text { Temperature } \\
\left(\mathrm{MK}^{\circ}\right)\end{array}$ & $\begin{array}{c}\text { Temperature } \\
\left(\mathrm{MK}^{\circ}\right)\end{array}$ & $\begin{array}{c}\text { Temperature } \\
\left(\mathrm{MK}^{\circ}\right)\end{array}$ \\
$c_{s} / v_{\text {esc }}$ & $\begin{array}{c}M_{*}=1 M_{\odot} \\
R_{*}=1 R_{\odot}\end{array}$ & $\begin{array}{c}M_{*}=0.7 M_{\odot} \\
R_{*}=0.65 R_{\odot}\end{array}$ & $\begin{array}{c}M_{*}=0.5 M_{\odot} \\
R_{*}=0.47 R_{\odot}\end{array}$ & $\begin{array}{c}M_{*}=0.2 M_{\odot} \\
R_{*}=0.22 R_{\odot}\end{array}$ \\
\hline 0.2219 & 1.30 & 1.40 & 1.39 & 1.20 \\
0.25 & 1.65 & 1.77 & 1.77 & 1.52 \\
0.33 & 2.88 & 3.09 & 3.08 & 2.66 \\
0.4 & 4.23 & 4.53 & 4.53 & 3.90 \\
\hline
\end{tabular}

polytropic index, $\gamma$. In this study, we focus on how different coronal temperatures affect the driving of the outflow. The following three dimensionless velocities are the main input parameters of our initial setup: the ratio of the adiabatic sound speed, defined at the stellar surface, to the escape speed, $c_{s} / v_{\text {esc }}$, where $c_{s}=\sqrt{\gamma p_{*} / \rho_{*}}$ (the "** denotes values at $R_{*}$ ) and $v_{\text {esc }}=\sqrt{2 G M_{*} / R_{*}}$; the ratio of the Alfvén speed to the escape speed, $v_{\mathrm{A}} / v_{\mathrm{esc}}$, where $v_{\mathrm{A}}=B_{*} / \sqrt{4 \pi \rho_{*}}$; and the stellar spin rate, $f$, which is the ratio of the stellar equatorial rotation velocity to the break-up speed, where the break-up speed is $v_{\text {kep }}=v_{\text {esc }} / \sqrt{2}$. The latter will be held fixed for our study close to the solar value, $f=0.00393$. The polytropic index $\gamma$ and the magnetic field geometry are also parameters, but we only vary the dipolar field strengths and we fix $\gamma=1.05$ (Washimi \& Shibata 1993; Matt et al. 2012; Réville et al. 2015a), which behaves like an adiabatically expanding flow that has energy input as the wind expands, such that $p \propto \rho^{1.05}$.

A polytropic treatment of the outflow acceleration is suitable for our purpose because we do not attempt to produce stellar wind solutions that will exhibit plasma properties similar to the ones observed in the solar wind such as speed bimodality, temperature contrast, and density between coronal holes and helmet streamers. Regardless, studies have shown that the polytropic approximation can capture the large-scale structure of the solar corona magnetic field (see, e.g., Mikić et al. 1999; Riley et al. 2006) and produces wind solutions with velocity profiles that agree with the observed solar wind on large scales (see, e.g., Keppens \& Goedbloed 1999; Ofman 2004).

Using the ideal-gas equation of state, the stellar coronal temperature can be written in terms of the parameter $c_{s} / v_{\mathrm{esc}}$,

$$
T_{*}=\left(\frac{c_{s}}{v_{\mathrm{esc}}}\right)^{2}\left(\frac{2 G M_{*} \tilde{\mu} m_{p}}{\gamma R_{*} k_{\mathrm{B}}}\right),
$$

where $k_{\mathrm{B}}$ is the Boltzmann constant, $m_{p}$ is the proton mass, and $\tilde{\mu}$ is the mean atomic weight (i.e., the average mass per particle measured in units of $m_{p}$ ). For given stellar parameters, the temperature depends on the mean atomic weight, $\tilde{\mu}$, which is determined by the chemical composition and the atomic physics of the stellar atmosphere. For a solar coronal plasma, $\tilde{\mu}=0.6$ (e.g., Priest 2014), Table 1 translates $c_{s} / v_{\text {esc }}$ into Kelvin for solar parameters (with $M_{\odot}=1.99 \times 10^{33} \mathrm{~g}$ and $R_{\odot}=6.96 \times 10^{10} \mathrm{~cm}$ ) and for stars at the age of the Sun, with parameters of $M_{*}=0.7,0.5,0.2 M_{\odot}$, and respectively, $R_{*}=0.65,0.47,0.22 R_{\odot}$, taken from the stellar evolution models of Baraffe et al. (1998).

Figure 1 shows the velocity profiles of the polytropic models for different coronal temperatures, represented in the plot by 
Table 2

Simulation Input Parameters and the Resulting Global Wind Properties

\begin{tabular}{|c|c|c|c|c|c|c|c|}
\hline Case & $c_{S} / v_{\mathrm{esc}}$ & $v_{\mathrm{A}} / v_{\mathrm{esc}}$ & $\Upsilon$ & $\left\langle R_{\mathrm{A}}\right\rangle / R_{*}$ & $\Upsilon_{\text {open }}$ & $\Phi_{\text {open }} / \Phi_{*}$ & $\overline{\bar{V}_{R_{\mathrm{A}}} / v_{\mathrm{esc}}}$ \\
\hline 1 & 0.2219 & 0.0151 & 2.90 & 3.62 & 283 & 0.787 & 0.0567 \\
\hline 2 & 0.2219 & 0.0301 & 11.9 & 5.52 & 1020 & 0.737 & 0.128 \\
\hline 3 & 0.2219 & 0.0452 & 27.7 & 6.23 & 1470 & 0.581 & 0.146 \\
\hline 4 & 0.2219 & 0.0753 & 79.9 & 7.27 & 2330 & 0.430 & 0.17 \\
\hline 5 & 0.2219 & 0.105 & 157 & 8.07 & 3170 & 0.358 & 0.187 \\
\hline 6 & 0.2219 & 0.301 & 1240 & 11.8 & 9810 & 0.224 & 0.264 \\
\hline 7 & 0.2219 & 0.627 & 5980 & 16.5 & 25600 & 0.165 & 0.335 \\
\hline 8 & 0.2219 & 0.953 & 15000 & 20.2 & 44300 & 0.137 & 0.374 \\
\hline 9 & 0.2219 & 1.51 & 41200 & 25.3 & 81300 & 0.112 & 0.415 \\
\hline 10 & 0.25 & 0.21 & 33.2 & 4.71 & 1170 & 0.473 & 0.206 \\
\hline 11 & 0.25 & 0.301 & 69.1 & 5.47 & 1820 & 0.409 & 0.236 \\
\hline 12 & 0.25 & 0.627 & 335 & 7.83 & 5070 & 0.309 & 0.312 \\
\hline 13 & 0.25 & 0.953 & 899 & 9.83 & 9460 & 0.258 & 0.361 \\
\hline 14 & 0.25 & 1.51 & 2720 & 12.7 & 18600 & 0.208 & 0.413 \\
\hline 15 & 0.25 & 2.5 & 8990 & 16.8 & 38200 & 0.164 & 0.465 \\
\hline 16 & 0.25 & 4.14 & 29100 & 22.0 & 75700 & 0.128 & 0.512 \\
\hline 17 & 0.33 & 0.953 & 16.7 & 3.27 & 1300 & 0.704 & 0.453 \\
\hline 18 & 0.33 & 2.5 & 173 & 5.79 & 5470 & 0.448 & 0.609 \\
\hline 19 & 0.33 & 3.01 & 275 & 6.47 & 7180 & 0.406 & 0.639 \\
\hline 20 & 0.33 & 4.14 & 612 & 7.86 & 11400 & 0.344 & 0.683 \\
\hline 21 & 0.33 & 6.2 & 1650 & 10.1 & 20700 & 0.282 & 0.736 \\
\hline 22 & 0.33 & 11 & 6630 & 14.3 & 45600 & 0.209 & 0.802 \\
\hline 23 & 0.33 & 17.5 & 20500 & 18.6 & 85000 & 0.162 & 0.845 \\
\hline 24 & 0.4 & 4.14 & 194 & 5.68 & 7900 & 0.507 & 0.904 \\
\hline 25 & 0.4 & 6.2 & 505 & 7.27 & 13800 & 0.416 & 0.969 \\
\hline 26 & 0.4 & 8.6 & 1090 & 8.76 & 20800 & 0.348 & 1.01 \\
\hline 27 & 0.4 & 11 & 1960 & 10.2 & 28800 & 0.305 & 1.04 \\
\hline 28 & 0.4 & 17.5 & 5890 & 13.0 & 50800 & 0.234 & 1.10 \\
\hline 29 & 0.4 & 26 & 13700 & 16.4 & 90400 & 0.204 & 1.14 \\
\hline 30 & 0.4 & 50 & 62700 & 22.7 & 193000 & 0.140 & 1.21 \\
\hline
\end{tabular}

the dimensionless quantity $c_{s} / v_{\text {esc }}$. Each curve in this plot is the analytic solution of the wind speed as a function of radial distance from the stellar surface, and each temperature is indicated by a different color. The plot shows that a hotter wind starts on the stellar surface at a higher speed and also reaches a higher terminal speed. To be more specific, for this range in $c_{s} / v_{\text {esc }}$, the flow speed varies by 3.5 orders of magnitude at $R_{*}$, and by more than a factor of 2 at $50 R_{*}$. Moreover, a hotter wind accelerates more rapidly compared to a cooler wind, meaning that at every radius, the hotter wind exhibits a higher value of both $d v_{r} / d t$ and $d v_{r} / d r$. The input parameter $c_{s} / v_{\text {esc }}$ varies between 0.2219 and 0.4 , a range that was selected to produce reasonable wind velocity profiles for the whole grid of simulations, for a given polytropic index (i.e., $\gamma=1.05$ in our case). This range ensures that the lowest temperature still results in a high enough flow terminal velocity for the wind to be able to escape the star's gravity field. The upper limit for our flow temperature is determined so that it initiates at the stellar corona at subsonic velocities. Our wind solution with $c_{s} / v_{\text {esc }}=0.4$ starts at the bottom of the flow with an initial speed that is already $50 \%$ of the sound speed, defined at the stellar surface (see Figure 1), and the wind becomes supersonic at $r=1.7 R_{*}$. Higher temperatures will result in outflows with unrealistically high base velocities (i.e., almost supersonic flow at the stellar surface). Although the polytropic wind formalism includes simplified physics that do not incorporate all relevant coronal processes that drive such outflows, Figure 1 shows that the range of winds we consider in our study covers a wide range of wind acceleration profiles, which may encompass the range of velocities encountered in real stellar winds under various coronal conditions.

Table 2 presents the parameters varied (second and third columns) for all of the simulated wind cases in the study. The magnetization of the wind is computed using the formula introduced in Matt \& Pudritz (2008),

$$
\Upsilon \equiv \frac{B_{*}^{2} R_{*}^{2}}{\dot{M}_{w} v_{\mathrm{esc}}},
$$

and the quantity $\Upsilon$ can be regarded as the ratio of the magnetic field energy to the kinetic energy of the flow, or as representing the interplay between the Lorentz forces and the inertia of the wind (ud-Doula \& Owocki 2002). In Equation (11), $\dot{M}_{w}$ is extracted directly from the simulations, and, for a given surface density, depends on the wind-driving physics, the magnetic field structure/configuration, and the numerical setup (for further discussions, see Matt et al. 2012 and Section 4.1). Therefore, we choose to present $\Upsilon$ as the second independent variable of the study, even though $v_{\mathrm{A}} / v_{\mathrm{esc}}$ is the input parameter that controls the magnetic field strength. All the values of $\Upsilon$ are listed in the fourth column in Table 2. The parameter space that has been explored during the entire study is visualized in Figure 2, and each simulation is one symbol in this plot. The different symbols and their corresponding colors represent cases with different temperatures, and overall, we covered three to four orders of magnitude in wind magnetization for each temperature. 


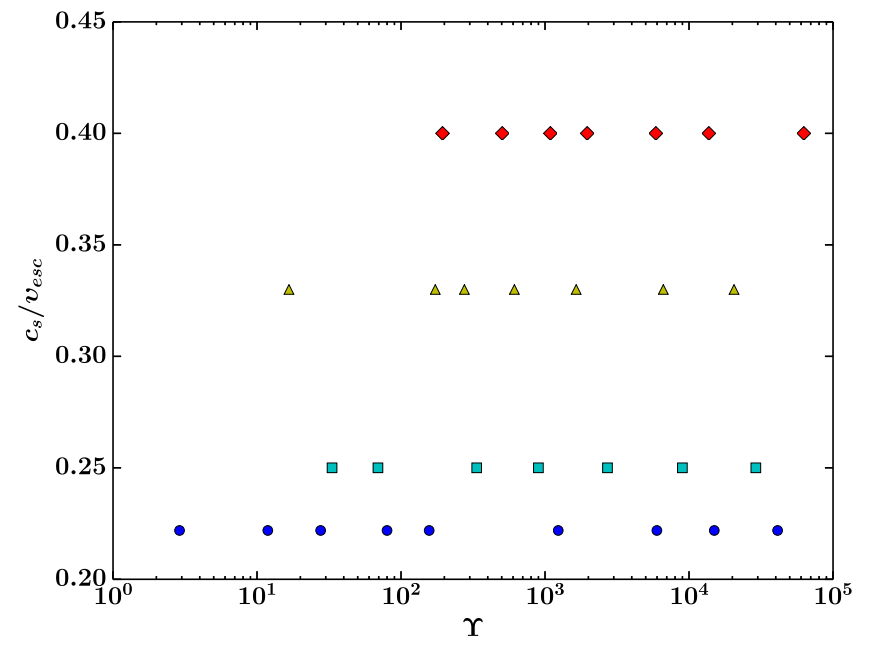

Figure 2. Parameter space for the 30 simulations in this study. The vertical axis shows the parameter $c_{s} / v_{\mathrm{esc}}$, which controls the flow temperature. The horizontal axis shows the parameter $\Upsilon$, which is the wind magnetization (see Equation (11)), and is associated with the average, stellar-surface magnetic field strength. Circles (blue), squares (cyan), triangles (yellow), and diamonds (red) correspond to simulations with $c_{s} / v_{\mathrm{esc}}=0 . .219,0.25,0.33$, and 0.4 , respectively. Every symbol represents a single case for which we have a steady-state wind solution.

\subsection{Wind Velocity Profiles}

At the start of a simulation, the presence of rotation and magnetic field modifies the initial, spherical symmetric flow, but after some period of evolution, the solution relaxes to a steady state. In order to highlight the influence of the gas temperature on the wind speed in our 2.5D MHD simulations, Figure 3 shows the flow poloidal velocity as a colorscale on a subset of our domain for two steady-sate wind solutions. Both cases shown have the same order of magnitude in parameter $\Upsilon$. The sonic surface is denoted by $R_{s}$ (dashed line) and the Alfvénic surface by $R_{A}$ (dotted-dashed line). Open-field lines, which correspond to wind streamlines, are also shown. A higher coronal temperature increases the velocity of the flow (bottom panel), and as a result, the sonic surface is closer to the stellar surface. The location of the Alfvén surface also comes closer to the star, and this is due to a hotter and faster wind, and also to a slightly lower magnetization of that case (i.e., case 13) relative to the top panel case (i.e., case 6).

To show how the wind velocity profile varies with latitude, Figure 4 illustrates the poloidal speed versus radial distance of the plasma flowing along the streamlines of the two cases shown in Figure 3. Each velocity law in Figure 4 is individually colored and matches the colors of the open-field lines plotted in Figure 3 . The streamlines were chosen to be at various latitudes at $50 R_{*}$. The plot comprises two groups of lines, one for each case, and the upper set corresponds to the hotter wind (i.e., case 13). Once more, it is clear that the hotter wind accelerates more rapidly and is faster everywhere. An interesting feature shown in Figure 4 is that each field line produces a unique velocity profile. This behavior should be attributed to the different geometric expansion of flux tubes near the pole and close to the equator, something that was originally pointed out in Pneuman \& Kopp (1971). The fact that the 2D wind speed profiles are always faster compared to their 1D hydrodynamic counterparts (black dashed lines) occurs because of the overall, faster-than- $r^{2}$ divergence (i.e.,

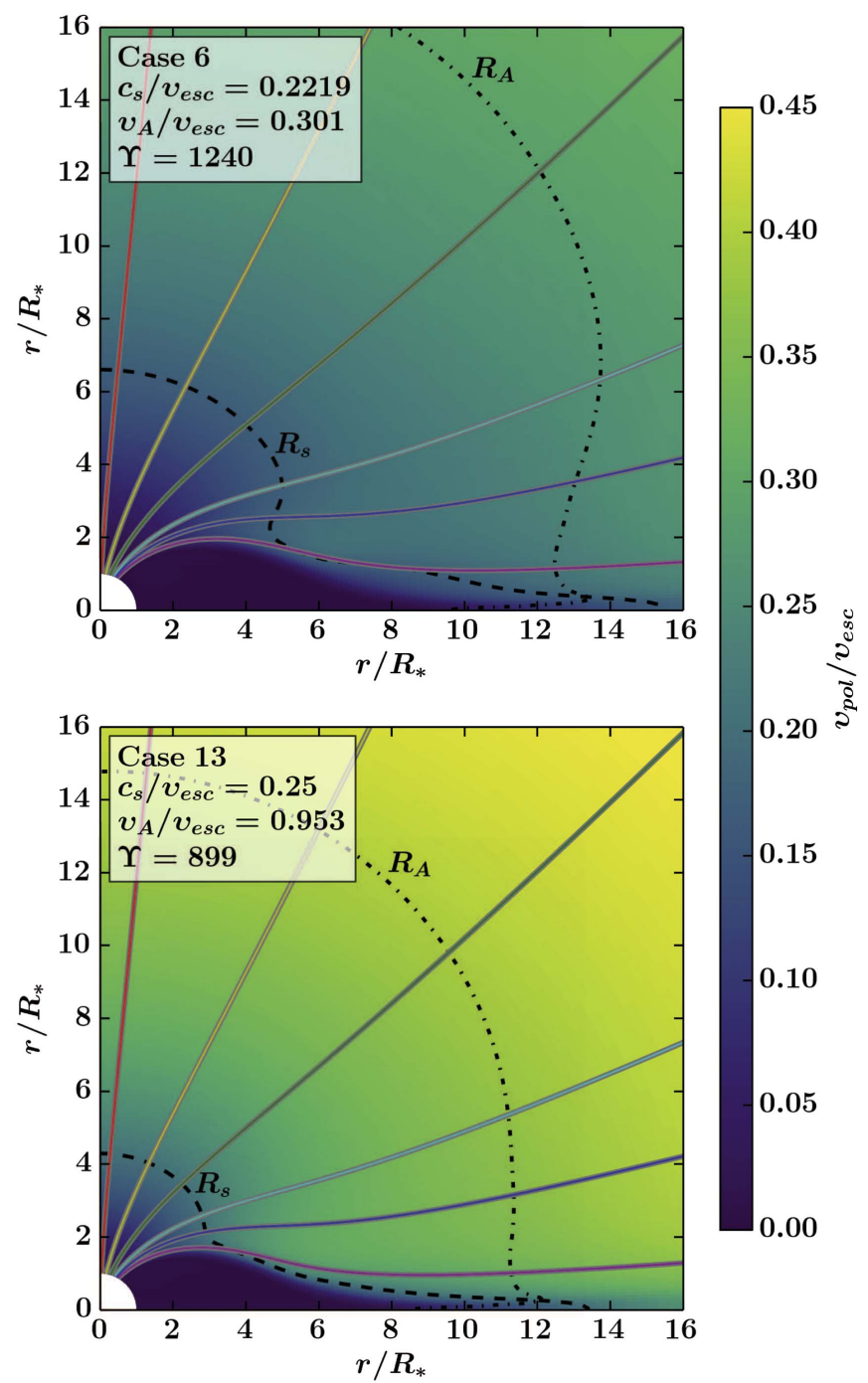

Figure 3. Poloidal velocity (colorscale) with magnetic field lines, for two steady-state wind solutions of this study that demonstrate the two-dimensional structure of the wind and the effect of the temperature on flows with similar magnetization (parameter $\Upsilon$ ) values. The dashed lines depict the sonic surface, and the dotted lines depict the Alfvénic surface. Each field line is plotted with a different color to indicate the paths along the flow open streamers, plotted in Figure 4. The images show only the northern stellar hemisphere and the inner portion of the whole computational domain.

superradial expansion) of the field geometry that channels the flow (e.g., Pneuman 1966; Kopp \& Holzer 1976; Réville et al. 2016b). Since all of our models are in the slow-magneticrotator regime (Belcher \& MacGregor 1976), magnetocentrifugal effects are negligible. We verified that in the absence of rotation, wind speed profiles do not change by more than $2 \%$ compared with simulated cases from rotating stars. Furthermore, we observed that, everything else being equal, an increase in the surface field strength will produce a wind solution that is faster everywhere (by $\sim 10 \%$ ), also due to a different geometrical expansion of the flux tubes' cross-section. The circles in Figure 4 repsresent the location of the local Alfvén radius, the radial distance at which the flow along each field line reaches the local poloidal Alfvén speed. From the stellar pole to the equator, the spherical Alfvén radius decreases because the Alfvénic surface reaches the cusp or neutral point of the helmet streamer (closed magnetic loops), which 


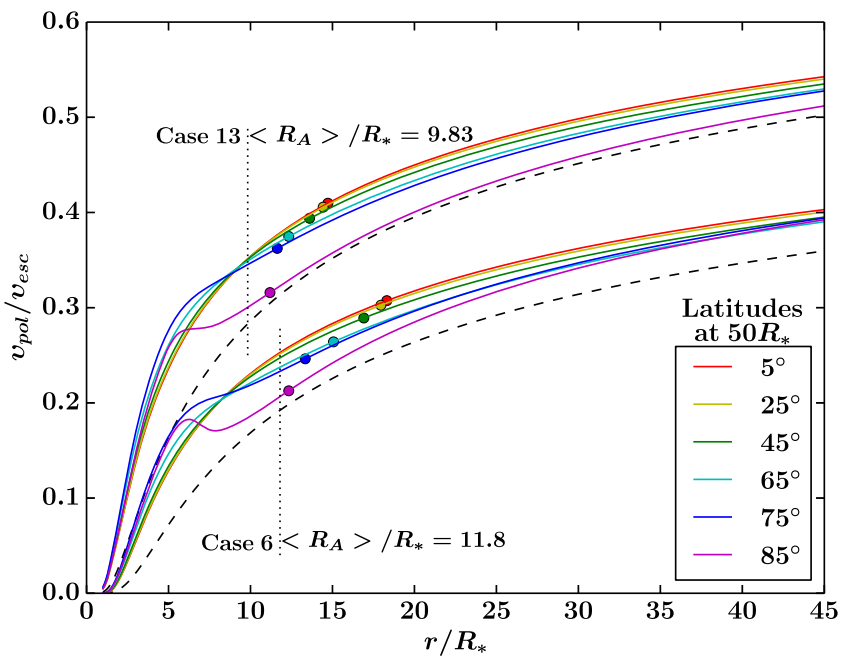

Figure 4. Wind speed profiles along open-field lines at different latitudes, as a function of radial distance, for the cases shown in Figure 3. Each line color correlates with the plotted field lines in Figure 3. For comparison, the dashed lines represent the velocity profiles of pure, one-dimensional hydrodynamic winds. The dotted lines show the torque-averaged Alfvén radius or magnetic lever arm of the magnetized outflow.

determines the transition region from sub-Alfvénic to superAlfvénic flows for streamers adjacent to the last closed-field line (Pneuman \& Kopp 1971). Finally, the black dotted vertical lines depict the size of the effective Alfvén radius. The local $R_{A}$ in each streamline is always larger compared to $\left\langle R_{\mathrm{A}}\right\rangle$ because the latter represents a mean value of the cylindrical Alfvén radius. Comparing the two cases, simulation 13 has a smaller effective lever arm, due to both a higher coronal temperature and a smaller $\Upsilon$ value, and this yields a less efficient braking torque on the star.

\section{Global Stellar Wind Properties}

\subsection{Mass and Angular-Momentum Outflow Rates}

Figure 5 displays the colorscale plots of the logarithmic density with velocity vectors and magnetic field lines (white lines) for four steady-state wind solutions of our study. Each case in Figure 5 has the same order of magnitude (and about the same value) in magnetization but a different plasma temperature. Qualitatively, we identify that hotter winds lead to both a smaller sonic surface (blue line) and Alfvénic surface (cyan line) as a consequence of being faster everywhere in the grid.

The global outflow rates of mass, $\dot{M}_{w}$, and angular momentum, $\tau_{w}$, are numerically computed for each steadystate wind solution of the study by using

$$
\begin{aligned}
& \dot{M}_{w}=\oint_{S} \rho \boldsymbol{v} \cdot d \boldsymbol{S}, \\
& \tau_{w}=\oint_{S} \Lambda \rho \boldsymbol{v} \cdot d \boldsymbol{S},
\end{aligned}
$$

where the integration occurs over any spherical surface that encloses the star within our computational domain, and

$$
\Lambda=r \sin \theta\left(v_{\phi}-B_{\phi} \frac{B_{p}}{\rho v_{p}}\right) .
$$

In the ideal MHD regime, $\Lambda$ gives the specific angular momentum carried away by the wind along a streamline and is a constant of motion for an axisymmetric, steady-state flow. In practice, we calculate both rates as functions of the spherical radius $r$ and use the median values obtained from all of the integrated $\dot{M}_{w}(r)$ and $\tau_{w}(r)$ over spherical shells above $10 R_{*}$ as the global $\dot{M}_{w}$ and $\tau_{w}$. This method avoids numerical diffusion effects that might cause non-conservation of mass and angularmomentum flux close to the stellar boundary. We then determine the torque-averaged Alfvén radius, $\left\langle R_{\mathrm{A}}\right\rangle / R_{*}$, from Equation (2), and these are listed in the fifth column of Table 2.

Another way to illustrate the range of the parameter space is to express $\Upsilon$ in terms of the input parameter $v_{\mathrm{A}} / v_{\mathrm{esc}}$. By manipulating Equation (11), one can derive that $\Upsilon \propto\left(v_{\mathrm{A}} / v_{\text {esc }}\right)^{2} / \dot{M}_{w}$ (i.e., $\Upsilon$ depends on $v_{\mathrm{A}} / v_{\text {esc }}$, which controls the surface magnetic field strength, but is also inversely proportional to the stellar mass-loss rate, which is an output of the simulations). Figure 6 shows that the four different temperatures of our models follow four different scaling laws for $\Upsilon$ versus the square of $v_{\mathrm{A}} / v_{\text {esc }}$. An increase in $c_{s} / v_{\text {esc }}$ significantly affects the stellar mass-loss rates by increasing the speed at the base of the wind. As a consequence, $\Upsilon$ decreases, and therefore we altered the range of the field strength (i.e., variation in $v_{\mathrm{A}} / v_{\text {esc }}$ ) for each temperature in order to achieve about the same range in the wind magnetization for all temperatures. By doing this, we avoid simulations with a small value of $v_{\mathrm{A}} / v_{\mathrm{esc}}$, and as a consequence, a small value of $\Upsilon$, since for such cases the Alfvén surface is very close to the stellar surface. There is no physical reason for not considering cases with wind magnetization above $10^{5}$, but these simulations start to become numerically very challenging, due to the smaller numerical time steps and large numerical errors (for further details on the accuracy of the numerical solutions, see Appendix B).

The gray lines in Figure 6 correspond to scaling laws with slopes of unity and show how the parameter $\Upsilon$ would depend on $v_{\mathrm{A}} / v_{\mathrm{esc}}$ if the stellar mass-loss rate were constant for a grid of simulations with a given coronal temperature, and thus independent of the stellar surface magnetic field strength. The fact that we find steeper power laws (the slopes are respectively 1.03, 1.14, 1.22 , and 1.16 for $c_{s} / v_{\text {esc }}=0.2219,0.25,0.33$, and 0.4 ) indicates that the mass-loss rates actually decrease with increased field strengths. This feature can be physically explained by an interplay between two competing effects. A stronger field leads to a slightly faster flow (discussed in Section 3.3), but also to a smaller area on the stellar surface carrying mass flow. Figure 6 indicates that the net result is a slightly decreasing $\dot{M}_{w}$. A similar trend was also seen in Réville et al. (2015a). Nevertheless, we should be cautious in interpreting the scaling laws in Figure 6 as realistic stellar mass-loss indicators, since polytropic wind models lack the exact physics that drive outflows from solar- and late-type stars. Early studies on the solar wind (Leer \& Holzer 1980) showed that where energy is added in the flow has a big influence on the resulting solar mass-loss rate. Moreover, the latest theoretical models (Cranmer \& Saar 2011; Suzuki et al. 2013) suggest that a realistic treatment of coronal heating is needed for accurate predictions on the stellar mass-loss rates from cool stars. Therefore, the scaling laws between $\Upsilon$ and $v_{\mathrm{A}} / v_{\mathrm{esc}}$ can be interpreted as part of the generic phenomenology in our simulations and should not be regarded as trends that give accurate predictions on mass-loss rates in solar- and late-type stars. Still, our formulae should provide the exerted magnetic torque for any given $\dot{M}_{w}$, extracted from observations (e.g., Wood 

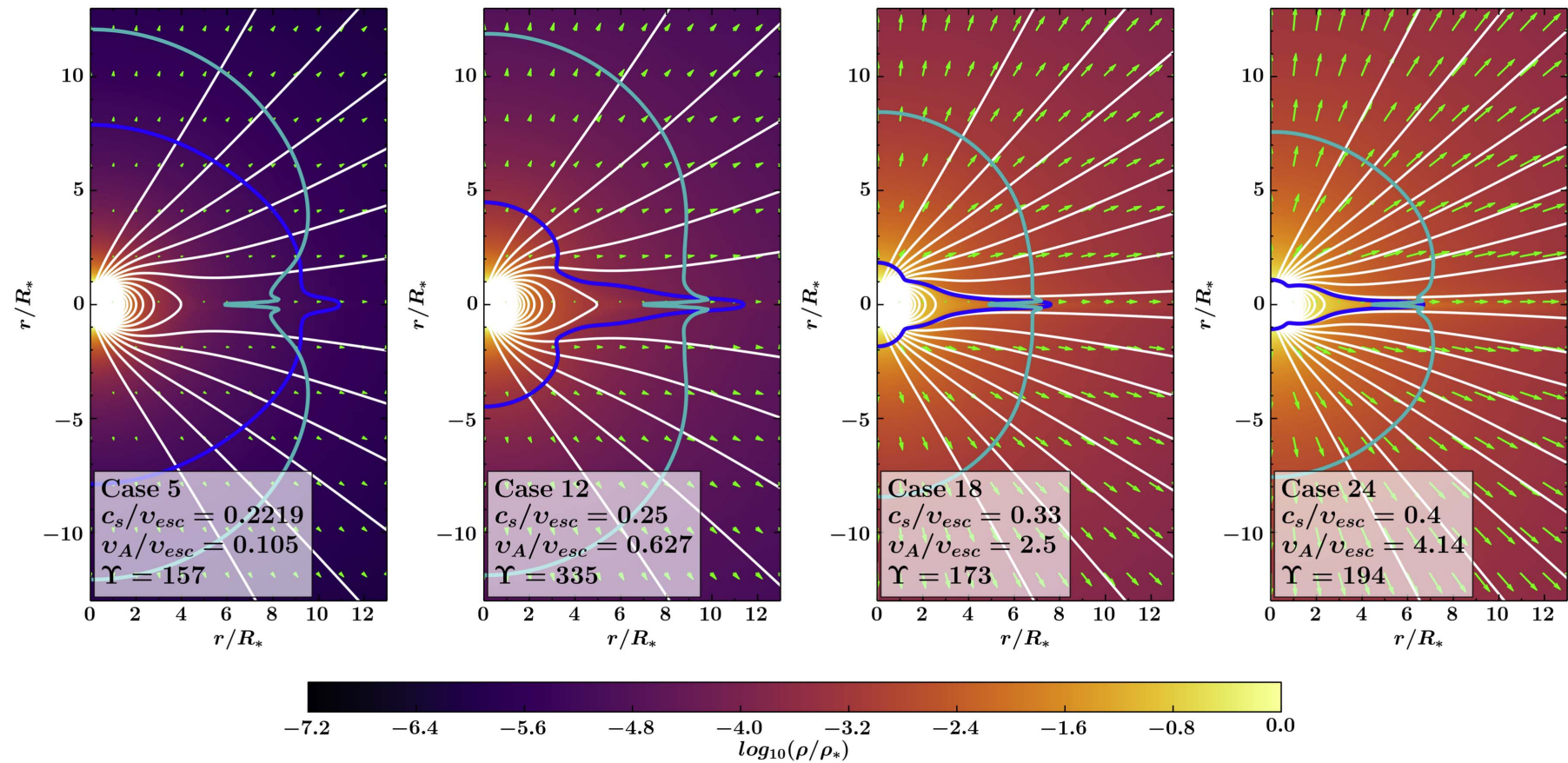

Figure 5. Color maps of the logarithmic density, magnetic field lines, and velocity vectors in the inner region of the four simulations with similar magnetization, $\Upsilon$, but varying wind temperature (characterized by $c_{s} / v_{\mathrm{esc}}$ ). The blue and cyan lines show the location of the sonic and Alfvénic surfaces, respectively. A higher surface plasma temperature, for about the same value of $\Upsilon$, results in a denser wind and the two critical surfaces being closer to the star.

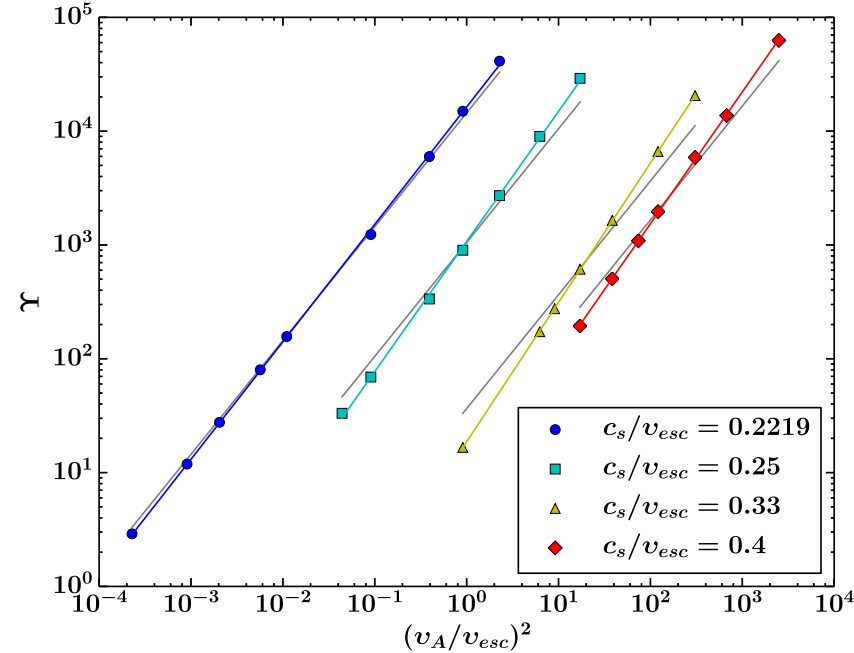

Figure 6. Wind magnetization, $\Upsilon$, vs. the square of the input parameter $v_{\mathrm{A}} / v_{\text {esc }}$. The same colors/symbols correspond to a grid of simulations with the same value of $c_{s} / v_{\mathrm{esc}}$ (as in Figure 2). In our simulations, $\Upsilon \propto\left(v_{\mathrm{A}} / v_{\mathrm{esc}}\right)^{2} / \dot{M}_{w}$, and for a given value of $v_{\mathrm{A}} / v_{\mathrm{esc}}$, a hotter wind has a much higher mass-loss rate. Gray scaling laws have a slope of unity. For a given coronal temperature, each scaling law has a slope steeper than unity, indicating that $\dot{M}_{w}$ decreases weakly with increasing $v_{\mathrm{A}} / v_{\text {esc }}$.

et al. 2002, 2014) or modeling (e.g., Holzwarth \& Jardine 2007; Cranmer \& Saar 2011; Suzuki et al. 2013).

\subsection{Scaling Laws between the Alfvén Radius and $\Upsilon$}

The dependence of the effective Alfvén radius, $\left\langle R_{\mathrm{A}}\right\rangle / R_{*}$, on wind magnetization, $\Upsilon$, for all numerical solutions of the study is depicted in Figure 7. Each point in Figure 7 corresponds to a single simulation, and the color and symbols have the same

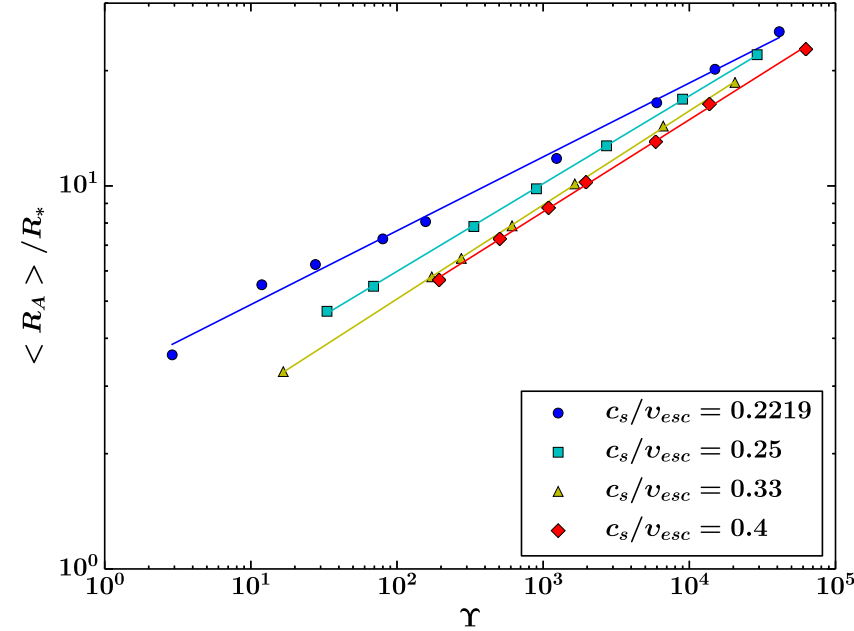

Figure 7. Dependence of the effective Alfvén radius, $\left\langle R_{\mathrm{A}}\right\rangle / R_{*}$, on $\Upsilon$ for all cases of the parameter study. The colors/symbols have the same meaning as in Figure 2. Four simple power laws of $\left\langle R_{\mathrm{A}}\right\rangle / R_{*}$ that depend on parameter $\Upsilon$ are shown, and each one corresponds to a different value of $c_{s} / v_{\text {esc }}$. For a given $\Upsilon$, the magnetic lever arm (i.e., $\left\langle R_{\mathrm{A}}\right\rangle / R_{*}$ ) of the wind decreases with increasing coronal temperature, and as a consequence, the torque exerted on the star becomes less efficient.

meaning as in Figure 6. In order to fit the simulation data, we use the formulation introduced in Matt \& Pudritz (2008), which scales $\left\langle R_{\mathrm{A}}\right\rangle / R_{*}$ as a power law in $\Upsilon$,

$$
\frac{\left\langle R_{\mathrm{A}}\right\rangle}{R_{*}}=K_{s} \Upsilon^{m_{s}},
$$

where $K_{s}$ and $m_{s}$ are dimensionless fitting constants, and Equation (15) determines $\left\langle R_{\mathrm{A}}\right\rangle / R_{*}$ in terms of the magnetic field strength on the stellar surface. Four different fitting laws 
Table 3

Fitting Constants ${ }^{\mathrm{a}}$ of the Parameter Study

\begin{tabular}{lcccccrrr}
\hline \hline$c_{s} / v_{\text {esc }}$ & $K_{s}$ & $m_{s}$ & $1 /(4+q)$ & $K_{o}$ & $m_{o}$ & $1 /(2+q)$ & $K_{q}$ \\
\hline 0.2219 & $3.1 \pm 0.1$ & $0.193 \pm 0.005$ & $0.202 \pm 0.004$ & $0.51 \pm 0.01$ & $0.343 \pm 0.003$ & $0.34 \pm 0.01$ & $0.023 \pm 0.005$ & $0.94 \pm 0.09$ \\
0.25 & $2.08 \pm 0.02$ & $0.229 \pm 0.001$ & $0.218 \pm 0.002$ & $0.34 \pm 0.01$ & $0.370 \pm 0.004$ & $0.386 \pm 0.006$ & $0.088 \pm 0.009$ & $0.59 \pm 0.04$ \\
0.33 & $1.64 \pm 0.01$ & $0.246 \pm 0.001$ & $0.230 \pm 0.002$ & $0.160 \pm 0.007$ & $0.418 \pm 0.004$ & $0.426 \pm 0.006$ & $0.32 \pm 0.02$ & $0.35 \pm 0.03$ \\
0.4 & $1.63 \pm 0.04$ & $0.240 \pm 0.003$ & $0.2378 \pm 0.0005$ & $0.118 \pm 0.006$ & $0.433 \pm 0.005$ & $0.454 \pm 0.002$ & $0.64 \pm 0.01$ & $0.205 \pm 0.009$ \\
& & & & & & & & \\
\hline $0.2219^{\mathrm{b}}$ & 2.49 & 0.2177 & $\ldots$ & $0.65 \pm 0.05$ & $0.31 \pm 0.02$ & 0.37 & $\ldots$ & $\ldots$ \\
$0.2219^{\mathrm{c}}$ & $2.0 \pm 0.1$ & $0.235 \pm 0.007$ & 0.21 & $\ldots$ & 0.7 \\
\hline
\end{tabular}

Notes.

${ }^{a}$ For Equations (15), (18), and (28) in Figures 7, 8, and 10, respectively.

b Matt et al. (2012).

${ }^{\mathrm{c}}$ Réville et al. (2015a, 2016a).

are shown in Figure 7, and the values of $K_{s}$ and $m_{s}$ for every fit are given in the second and third columns of Table 3.

Each value of $c_{s} / v_{\text {esc }}$ gives a simple power law of the torqueaveraged Alfvén radius on $\Upsilon$ for various surface magnetic field strengths. However, the fit parameters are different with each coronal temperature. The power law for $c_{s} / v_{\text {esc }}=0.2219$ is shallower (see also Table 3) compared with previous parameter studies (Matt et al. 2012; Réville et al. 2015a), and can be understood as an effect due to differences in the numerical setup between the studies (e.g., geometry of the problem, numerical scheme, different approaches to boundary conditions), indicative of systematic errors. Réville et al. (2015a) demonstrated that different power laws resulted from different field geometries. It was also shown that the complexity of the magnetic field does not significantly influence the wind acceleration. For this study, only dipolar fields are considered, but by varying the gas temperature, we actually change the acceleration of the flow. As a consequence, the wind speed also changes for simulations with different values of $c_{s} / v_{\mathrm{esc}}$, and that physically explains the four power laws in Figure 7. In conclusion, hotter winds are faster, and thus, the Alfvén surface comes closer to the star, the size of the lever arm or the effective Alfvén radius decreases, and therefore the magnetic braking torque that is exerted on the star becomes weaker.

\subsection{Scaling Laws Using the Amount of Open Magnetic Flux}

Réville et al. (2015a) introduced an alternative formulation for the torque-averaged Alfvén radius that scales $\left\langle R_{\mathrm{A}}\right\rangle / R_{*}$ as a power law in a new $\Upsilon$-like parameter that depends on the amount of open magnetic flux (see also Washimi \& Shibata 1993). In general, the unsigned magnetic flux of the stellar magnetic field, as a function of spherical radius $r$, can be evaluated as

$$
\Phi(r)=\oint_{S}|\boldsymbol{B} \cdot d \boldsymbol{S}|,
$$

where the integration is performed over spherical surfaces that enclose that star. For a given field geometry, a dipole in our case, the magnetic flux initially drops as $1 / r$, but there is a regime in which the thermal pressure and the inertia of the wind dominate over the magnetic stresses, the field completely

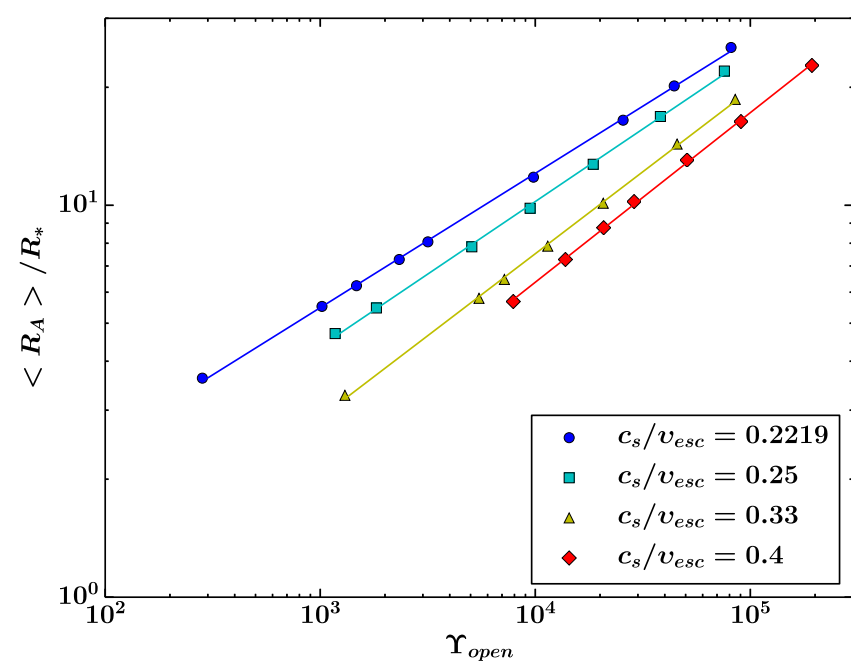

Figure 8. Effective Alfvén radius, $\left\langle R_{\mathrm{A}}\right\rangle / R_{*}$, vs. the parameter $\Upsilon_{\text {open }}$ (Equation (18)) for all of the simulations in the study. Colors/symbols are the same as in Figure 2. Four different fitting laws are shown, one for each set of wind solutions with a given value of $c_{s} / v_{\text {esc }}$. An increase in the temperature of the flow, for winds with the same value of $\Upsilon_{\text {open }}$, results in an increase in the size of $\left\langle R_{\mathrm{A}}\right\rangle / R_{*}$ and the efficiency of the braking torque.

opens, and the magnitude of the magnetic flux becomes constant (i.e., open magnetic flux); see, for example, Figure 5 in Réville et al. (2015a).

Following Réville et al. (2015a), the new $\Upsilon$-like parameter is defined as

$$
\Upsilon_{\mathrm{open}} \equiv \frac{\Phi_{\mathrm{open}}^{2}}{R_{*}^{2} \dot{M}_{w} v_{\mathrm{esc}}}
$$

where $\Phi_{\text {open }}$ is the open magnetic flux that is directly computed from the numerical simulations by Equation (16). For $\Phi_{\text {open }}$, for a given wind solution, we use the median value of $\Phi(r)$ above the corresponding $\left\langle R_{\mathrm{A}}\right\rangle / R_{*}$ of that solution, where we have identified that the magnetic flux is constant. The sixth column in Table 2 lists all of the values of $\Upsilon_{\text {open }}$. The seventh column in Table 2 contains all of the values of the fractional open flux 
(i.e., $\Phi_{\text {open }}$ normalized to the surface unsigned magnetic flux, $\left.\Phi_{*}\right)$, which can be written as $\Phi_{\text {open }} / \Phi_{*}=\left(\Upsilon_{\text {open }} / \Upsilon\right)^{1 / 2} /(4 \pi)$.

The value of $\left\langle R_{\mathrm{A}}\right\rangle / R_{*}$ versus the parameter $\Upsilon_{\text {open }}$, for the entire study, is presented in Figure 8. Similarly to Equation (15), a function in the form of

$$
\left\langle R_{\mathrm{A}}\right\rangle / R_{*}=K_{o} \Upsilon_{\text {open }}^{m_{o}}
$$

fits the data, and again $K_{o}$ and $m_{o}$ represent dimensionless fitting constants and $\left\langle R_{\mathrm{A}}\right\rangle / R_{*}$ is determined here in terms of the open magnetic flux. Four power laws are shown in Figure 8, and the fifth and sixth columns in Table 3 lists the values of the fitting constants for each scaling law. The figure demonstrates how the effective Alfvén radius scales as a simple braking law with the parameter $\Upsilon_{\text {open }}$, for every value of $c_{s} / v_{\text {esc }}$. Furthermore, Figure 8 reveals one of the key results in this parameter study. We show that the temperature of the flow, which affects the wind velocity and acceleration profile, is an important parameter in the magnetic-braking models. Réville et al. (2015a) showed that all of the wind solutions in their study followed one unique power law, demonstrating that the $\left\langle R_{\mathrm{A}}\right\rangle / R_{*}$ versus $\Upsilon_{\text {open }}$ scaling was independent of the field geometry, but they assumed a fixed stellar coronal temperature. The fact that our power law, for $c_{s} / v_{\mathrm{esc}}=0.2219$, is steeper (see also Table 3 ) compared to the single braking law found in Réville et al. (2015a) might be explained as an effect due to different choices of the numerical setups of the two studies, as discussed in the previous subsection. An influence on the braking laws, due to a different coronal temperature, has also been observed in Réville et al. (2016a). In conclusion, the temperature of the flow affects the size of the magnetic lever arm (i.e., $\left\langle R_{\mathrm{A}}\right\rangle / R_{*}$ ) and the efficiency of magnetic braking.

\section{Magnetic Braking Laws for Known Wind Acceleration Profiles}

\subsection{Semi-analytic Model for the Alfvén Radius versus $\Upsilon_{\text {open }}$}

We showed above that the flow temperature and the resulting wind acceleration can influence the efficiency of the braking toque. For this section, our objective is to provide a more generic braking law that will take this effect into account.

In order to mathematically express the dependence of the braking laws on the acceleration profile of the flow, we will employ a similar one-dimensional analysis to that used in earlier works (e.g., Kawaler 1988; Tout \& Pringle 1992; Matt \& Pudritz 2008; Réville et al. 2015a). For a one-dimensional MHD flow along a magnetic flux tube, the wind velocity at the Alfvén radius, by definition, is equal to the local Afvénic speed. This is

$$
v^{2}\left(R_{\mathrm{A}}\right)=v_{\mathrm{A}}^{2}=\frac{B_{\mathrm{A}}^{2}}{4 \pi \rho_{\mathrm{A}}},
$$

where $B_{A}$ and $\rho_{\mathrm{A}}$ are the local magnetic field and density, respectively, at the Alfvén surface. In order to evaluate $B_{A}$ at $R_{A}$, one must specify how the magnetic field strength depends on radius. Hence, for this work, we adopt a prescription similar to Mestel \& Spruit (1987; see also Mestel 1999), in which the magnetic field is approximated as having two regions. The inner region exists from the stellar surface out to the "open- field" radius, $R_{o}$, in which the field is a single power law in radius,

$$
B\left(r \leqslant R_{o}\right)=B_{*}\left(\frac{R_{*}}{r}\right)^{l+2},
$$

with $l=1$ for a dipole. The outer region lies above $R_{o}$ in which the field decreases as a monopole (i.e., $l=0$ ),

$$
B\left(r \geqslant R_{o}\right)=B_{o}\left(\frac{R_{o}}{r}\right)^{2},
$$

where $B_{o}$ denotes $B\left(R_{o}\right)$, given by Equation (20). We also assume that the flow is the same along every field line (i.e., all values are only a function of radius and not latitude) and in a steady state.

This treatment for the stellar magnetic field is a simple approximation for the real magnetic field configurations in a wind, where, near the star, the field closely resembles the potential field, and farther out, it is stretched to a nearly radial configuration by the flow (see, for example, Figure 5). For a detailed comparison of the magnetic field in a wind simulation with a potential and radial field, see Réville et al. (2015b).

In all of our simulations, the Alfvén surface is located at the open-field region, and therefore, we assume that the condition $R_{\mathrm{A}}>R_{o}$ holds for all our cases as if they were 1D flows. Then, by combining Equations (20) and (21), the magnetic field strength at $R_{A}$ can now be written as

$$
B_{\mathrm{A}}=B_{o}\left(\frac{R_{o}}{R_{\mathrm{A}}}\right)^{2}=B_{*}\left(\frac{R_{*}}{R_{o}}\right)^{l+2}\left(\frac{R_{o}}{R_{\mathrm{A}}}\right)^{2}
$$

Since the magnetic flux is conserved, it can be written at the Alfvén radius as

$$
\Phi_{\mathrm{A}}=4 \pi R_{\mathrm{A}}^{2} B_{\mathrm{A}}=4 \pi R_{o}^{2} B_{o}=\Phi_{\text {open }},
$$

which equals the total open flux in the wind. By combining Equations (17), (19), and (23), we get

$$
\left(\frac{R_{\mathrm{A}}}{R_{*}}\right)^{2}=\frac{1}{(4 \pi)^{2}} \Upsilon_{\text {open }} \frac{v_{\mathrm{esc}}}{v\left(R_{\mathrm{A}}\right)},
$$

where we used $\dot{M}_{w}=4 \pi \rho_{\mathrm{A}} R_{\mathrm{A}}^{2} v\left(R_{\mathrm{A}}\right)$ for a spherically symmetric flow in the open-field region.

Since our wind solutions are multidimensional, we can associate the terms $R_{\mathrm{A}} / R_{*}$ and $v\left(R_{\mathrm{A}}\right)$ in Equation (24) with the torque-averaged Alfvén radius, $\left\langle R_{\mathrm{A}}\right\rangle / R_{*}$, and $\bar{V}_{R_{\mathrm{A}}}$, where $\bar{V}_{R_{\mathrm{A}}}$ represents the average wind speed at the Alfvén surface. We define

$$
\bar{V}_{R_{\mathrm{A}}} \equiv \frac{\sum_{i}^{N} v\left[\left(R_{\mathrm{A}}\right)_{i}, \theta_{i}\right]}{N},
$$

where the sum is over each discretized grid point $i$ along the Alfvén surface. $\bar{V}_{R_{\mathrm{A}}}$ is computed individually for each case in the study, and the values are listed in the eighth column in Table 2 . 


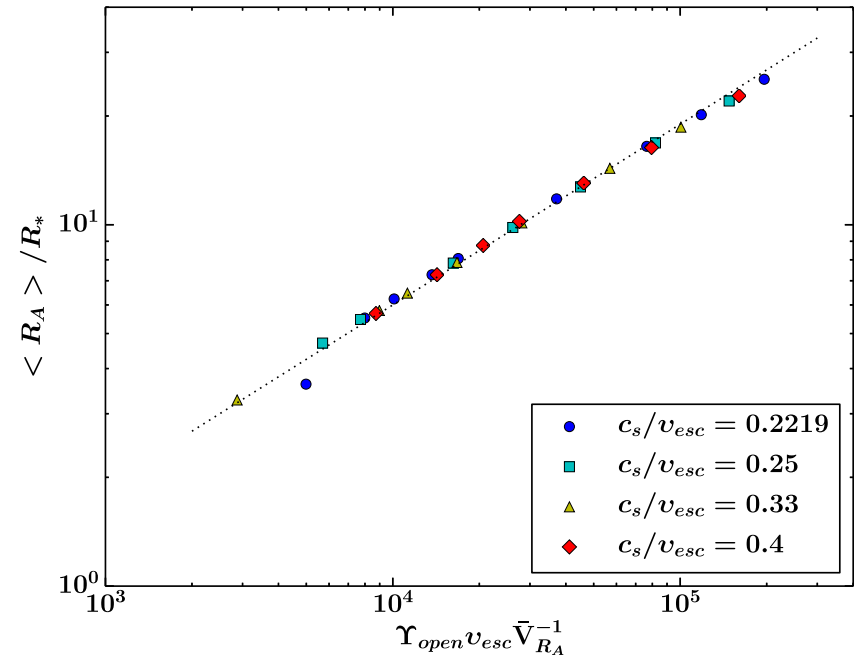

Figure 9. Effective Alfvén radius, $\left\langle R_{\mathrm{A}}\right\rangle / R_{*}$, vs. the quantity $\Upsilon_{\mathrm{open}} v_{\mathrm{esc}} \bar{V}_{R_{\mathrm{A}}}^{-1}$ for all of the simulation data. Colors/symbols have the same meaning as in Figure 2. All of the data points collapse into a single braking law, compared to Figure 8. The slope (or power-law index) of the dotted line is fixed to $1 / 2$ and fits the data according to Equation (26).

Following Equation (24), we plot $\left\langle R_{\mathrm{A}}\right\rangle / R_{*}$ versus the new quantity, $\Upsilon_{\text {open }} v_{\text {esc }} \bar{V}_{R_{\mathrm{A}}}^{-1}$, as depicted in Figure 9 , and fit the data to the function

$$
\frac{\left\langle R_{\mathrm{A}}\right\rangle}{R_{*}}=K_{c}\left(\Upsilon_{\text {open }} \frac{v_{\mathrm{esc}}}{\bar{V}_{R_{\mathrm{A}}}}\right)^{1 / 2},
$$

where again $K_{c}$ is introduced as a dimensionless fitting constant and its value should only deviate from $1 /(4 \pi)$ due to $2 \mathrm{D}$ effects, which were neglected in Equation (24). The best-fit value for $K_{c}$ gives

$$
K_{c}=0.7540\left(\frac{1}{4 \pi}\right) \pm 0.0004
$$

By including in our torque formalism the dimensionless term $v_{\text {esc }} / \bar{V}_{R_{\mathrm{A}}}$, which contains all of the information regarding the velocity and acceleration profile of the outflow, all of the data points in Figure 9 collapse into one single and precise power law. Hence, Equation (26) predicts the effective Alfvén radius of any wind, as long as $\bar{V}_{R_{\mathrm{A}}}$ and $\Upsilon_{\text {open }}$ are known.

\subsection{Power-law Approximation for the Wind Velocity at the Alfvén Radius, $\bar{V}_{R_{\mathrm{A}}}$}

Equation (26) can naturally explain the simple power laws in Figure 8 , if the wind speed, $\bar{V}_{R_{\mathrm{A}}}$, is also a power law in $\left\langle R_{\mathrm{A}}\right\rangle / R_{*}$, but with a scaling that varies for each temperature. To verify this, we plot $\bar{V}_{R_{\mathrm{A}}}$ versus the torque-averaged Alfvén radius, $\left\langle R_{\mathrm{A}}\right\rangle / R_{*}$, for all of the simulations in Figure 10. For comparison, the velocity profiles of the polytropic, Parker wind models, shown in Figure 1, are also plotted. We fit a power-law function to the data, given by

$$
\frac{\bar{V}_{R_{\mathrm{A}}}}{v_{\mathrm{esc}}}=K_{q}\left(\frac{\left\langle R_{\mathrm{A}}\right\rangle}{R_{*}}\right)^{q},
$$

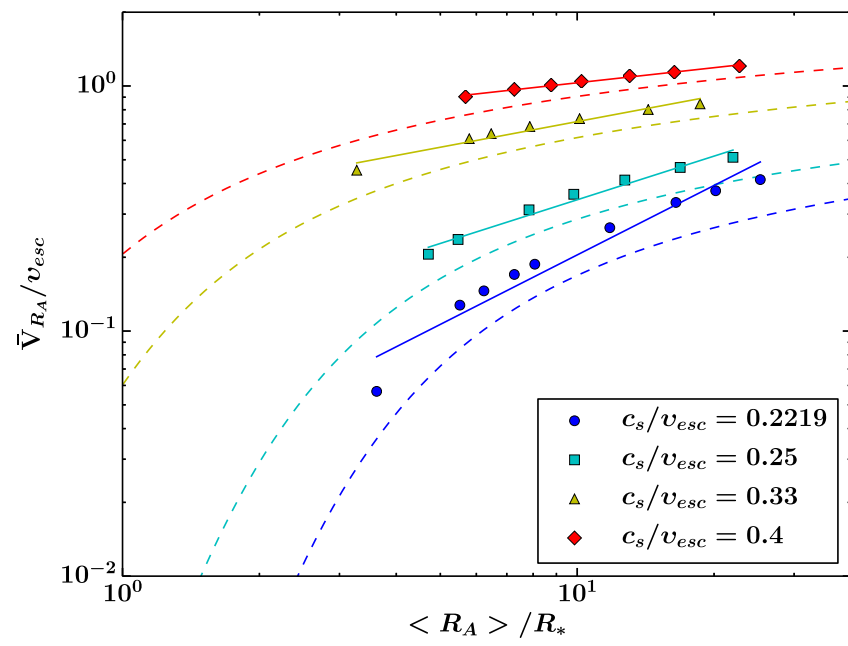

Figure 10. Average flow speed at the Alfvén surface, $\bar{V}_{R_{\mathrm{A}}}$, vs. $\left\langle R_{\mathrm{A}}\right\rangle / R_{*}$ for all of the simulated cases of the study. Colors/symbols are the same as in Figure 2. Each point in this plot represents the average wind speed at the Alfvén radius of a single wind solution (Equation (25)). The solid lines represent Equation (28) with the fit parameters listed in Table 3. For comparison, the dashed lines show the normalized radial velocity, $v_{r} / v_{\mathrm{esc}}$, as a function of the $r / R_{*}$ of the 1D hydrodynamic winds illustrated in Figure 1.

where $K_{q}$ and $q$ are both dimensionless fitting constants related to the acceleration profile of the wind. Each temperature gives us a separate pair of $K_{q}$ and $q$, tabulated in the eighth and ninth columns of Table 3, respectively. The value of $q$, found in Réville et al. (2015a), is also given in Table 3.

It is clear that Equation (28) is valid as a first-order approximation, despite the fact that the simulated winds do not follow a perfect power law (solid lines in Figure 10) and the behavior of $\bar{V}_{R_{\mathrm{A}}}$, as a function of $\left\langle R_{\mathrm{A}}\right\rangle / R_{*}$, exhibit a similar shape to the 1D hydrodynamic winds with the same value of $c_{s} / v_{\text {esc }}$ (dashed lines in Figure 10). Perhaps, for even more precise stellar-torque formulae, a different velocity law could be applied (e.g., modified beta law; see, for example, Lamers \& Cassinelli 1999). Nonetheless, over a small range of radii, these trends can be approximated by a power law, and that approximate fit, explains the power-law behavior in Figure 8. In addition, working with Equation (28), one can analytically solve Equation (26) for $\left\langle R_{\mathrm{A}}\right\rangle / R_{*}$ (see below).

Another interesting trend in Figure 10 is that the plotted data points are noticeably above the hydrodynamic wind velocity profiles. This can be understood as an effect due to both the differences in the dynamics of the two flows (i.e., MHD versus hydrodynamic flow) as discussed in Section 3.3, and the specific way the averaging and the scaling was done in Equation (28). Figure 10 also indicates why the braking laws in Figures 7 and 8 start to converge for higher coronal temperatures (e.g., the yellow and red lines with $\left.c_{s} / v_{\text {esc }}=0.33,0.4\right)$. Hotter flows enter the regime where the wind speed starts to saturate to wind terminal speed (i.e., speed at infinity) at a shorter radial distance compared to cooler winds. Hence, outflows that approach an almost constant speed suggest a $q$ that asymptotes to zero. Lastly, we found two empirical functions that predict the fitting constants $K_{q}$ and $q$ over any continuous range of values of $c_{s} / v_{\mathrm{esc}}$. These functions are

$$
K_{q}=1.36\left[5.87\left(c_{s} / v_{\mathrm{esc}}\right)^{2}-1.18\left(c_{s} / v_{\mathrm{esc}}\right)\right]
$$




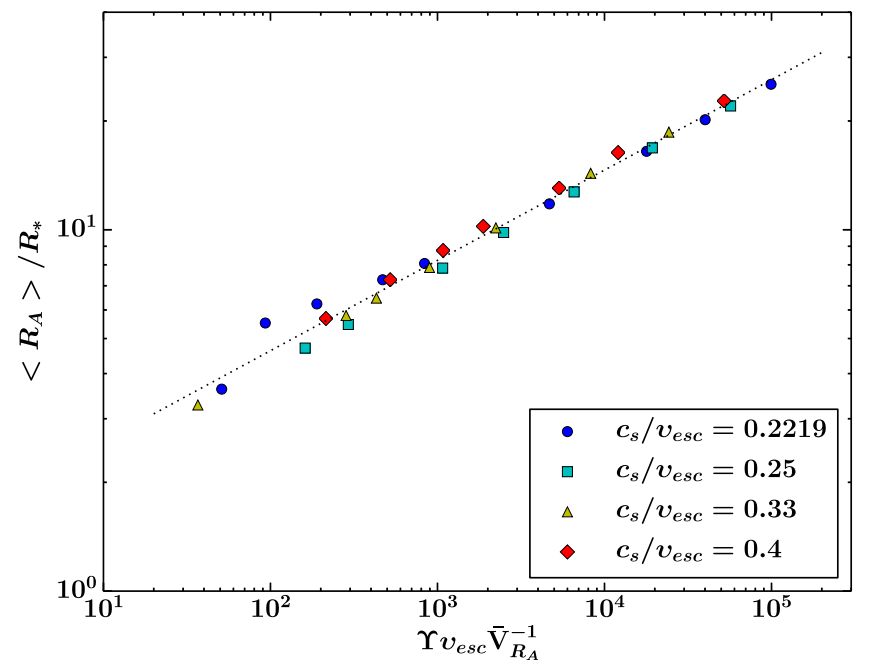

Figure 11. $\left\langle R_{\mathrm{A}}\right\rangle / R_{*}$ vs. the quantity $\Upsilon v_{\mathrm{esc}} \bar{V}_{R_{\mathrm{A}}}^{-1}$ for all simulations. Colors/ symbols have the same meaning as in Figure 2. All of the data points are fitted by a single coefficient $K_{l}$, and the fitting line (dotted line) has a slope (or powerlaw index) of 1/4, according to Equation (33). The small spread of the data points observed in this braking law is primarily due to variations in the ratio of the Alfvén radius to the open-field radius (see also Equation (32) and Figure 12).

$$
q=0.932\left[0.000979\left(c_{s} / v_{\mathrm{esc}}\right)^{-4.51}+0.553\left(c_{s} / v_{\mathrm{esc}}\right)\right] .
$$

The method and the derivation of Equations (29) and (30) are given in Appendix $\mathrm{C}$.

By combining Equations (26) and (28), we obtain

$$
\frac{\left\langle R_{\mathrm{A}}\right\rangle}{R_{*}}=\left(\frac{K_{c}^{2}}{K_{q}} \Upsilon_{\text {open }}\right)^{1 /(2+q)} .
$$

An interesting characteristic of Equation (31) is that it explains the fitting constants of Equation (18) in terms of the other fitting constants, and consists of an analytic expression for the effective Alfvén radius. This formalism is independent of the temperature of the flow (but requires a known wind acceleration profile) and the geometry of the magnetic field, and predicts the torque exerted on the star for any value of $\Upsilon_{\text {open }}$ for a given rotation rate (in the slow-rotator regime) and polytropic index $(\gamma=1.05$ in this study). Comparing Equations (18) and (31), we identify that $K_{o} \sim\left(K_{c}^{2} K_{q}^{-1}\right)^{1 /(2+q)}$ and $m_{o} \sim 1 /(2+q)$. The predicted values of $m_{o}$ for each temperature are listed in the seventh column of Table 3. Clearly, $m_{o}$ and $K_{o}$ strongly depend on the acceleration profile of the wind, here parametrized with $K_{q}$ and $q$.

\subsection{Semi-analytic Model for the Alfvén Radius Versus $\Upsilon$}

The formalism given by Equation (26) provides an excellent fit, in terms of predicting the torque-averaged Alfvén radius from the parameter $\Upsilon_{\text {open }}$, for a given wind acceleration. However, in real wind cases, the amount of open magnetic flux is a quantity that is not observable and can only be predicted (e.g., Vidotto et al. 2014b; Réville et al. 2015b; See et al. 2017a). Therefore, in this section, we aim to extract trends for the braking torque based on $\Upsilon$, which depends on the surface magnetic field strength (or surface magnetic flux).

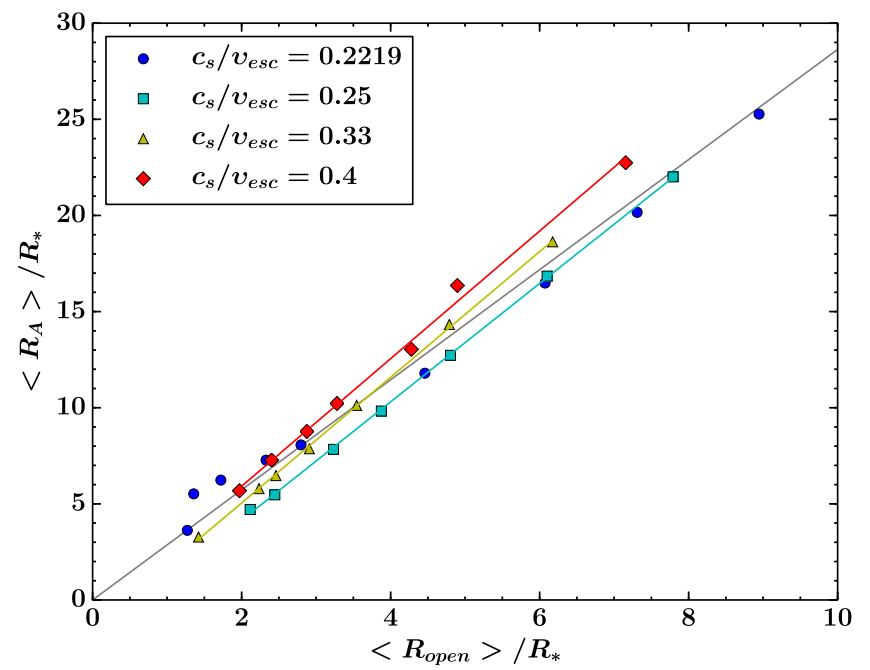

Figure 12. Torque-averaged Alfvén radius, $\left\langle R_{\mathrm{A}}\right\rangle / R_{*}$, vs. the normalized openfield radius, $\left\langle R_{o}\right\rangle / R_{*}$. Color/symbols are the same as in Figure 2. The gray line shows a linear function that represents all of the data and gives $\left\langle R_{\mathrm{A}}\right\rangle /\left\langle R_{o}\right\rangle=2.86$. The cyan, yellow, and red solid lines depict linear functions as well, for example, to show how $\left\langle R_{\mathrm{A}}\right\rangle /\left\langle R_{o}\right\rangle$ systematically varies for each temperature.

Such trends can be obtained analytically by combining Equations (22), (23), and (24), and also by using the definition for $\Upsilon$ (Equation (11)), which yields

$$
\left(\frac{R_{\mathrm{A}}}{R_{*}}\right)^{2 l+2}\left(\frac{R_{o}}{R_{\mathrm{A}}}\right)^{2 l}=\Upsilon \frac{v_{\mathrm{esc}}}{v\left(R_{\mathrm{A}}\right)} .
$$

Figure 11 shows the effective Alfvén radius versus the $\Upsilon$-based quantity $\Upsilon v_{\mathrm{esc}} \bar{V}_{R_{\mathrm{A}}}^{-1}$, as suggested by Equation (32). Once more, all of the details regarding the acceleration of the flow have been contained in the dimensionless term $v_{\text {esc }} / \bar{V}_{R_{\mathrm{A}}}$, and as a result, all of the simulations lie close to a single power law. By solving Equation (32) for $R_{\mathrm{A}} / R_{*}$, the power on this braking law depends only on the geometry of the field (or $l$ ). Hence, this should apply to more complex field geometries as well, but for our case with a dipole field $(l=1)$, the slope of the single line formed by the data points in Figure 11 is equal to $1 /$ 4. Following this simplified analysis, we fit the data in Figure 11 with

$$
\frac{\left\langle R_{\mathrm{A}}\right\rangle}{R_{*}}=K_{l}\left(\Upsilon \frac{v_{\mathrm{esc}}}{\bar{V}_{R_{\mathrm{A}}}}\right)^{1 / 4},
$$

and $K_{l}$ is introduced as the only fitting constant. The best-fit value of $K_{l}$ is

$$
K_{l}=1.46 \pm 0.02
$$

The fitting constant $K_{l}$ includes any factors due to the multidimensionality of our simulations, and most importantly, the comparison between Equations (32) and (33) suggests that $K_{l}$ also includes the dimensionless ratio of the Alfvén radius to the open-field radius of the wind, $R_{\mathrm{A}} / R_{o}$. Furthermore, the fact that all of the data points do not precisely lie along the single power law in Figure 11 implies that the term $R_{\mathrm{A}} / R_{o}$ is not constant for all of the simulations and exhibits a dependence on the flow temperature.

A coherent way to estimate the open-field radius (i.e., the radial distance in which the wind's thermal and ram pressure 
overpower the magnetic field pressure, and as a result the unsigned magnetic flux becomes constant as a function of radial distance) for all our simulations is to define $\left\langle R_{o}\right\rangle / R_{*}$ as

$$
\left(\frac{\left\langle R_{o}\right\rangle}{R_{*}}\right)^{l} \equiv \frac{\Phi_{*}}{\Phi_{\text {open }}} .
$$

In other words, Equation (35) gives the radial distance in which the function $\Phi(r) / \Phi_{*}=\left(R_{*} / r\right)^{l}$ intersects the line $\Phi(r) / \Phi_{*}=\Phi_{\text {open }} / \Phi_{*}=$ const. and applies for any given single magnetic field geometry.

In Figure 12, we present the normalized open-field radius, $\left\langle R_{o}\right\rangle / R_{*}$, versus the torque-averaged Alfvén radius, $\left\langle R_{\mathrm{A}}\right\rangle / R_{*}$, and the plot shows that all of the simulations have approximately the same ratio, $\left\langle R_{\mathrm{A}}\right\rangle /\left\langle R_{o}\right\rangle$. This feature explains why Equation (33) successfully represents the data. Assuming a linear scaling between $\left\langle R_{\mathrm{A}}\right\rangle$ and $\left\langle R_{o}\right\rangle$ yields $\left\langle R_{\mathrm{A}}\right\rangle /\left\langle R_{o}\right\rangle \approx 2.86$. A closer inspection reveals a range in $\left\langle R_{\mathrm{A}}\right\rangle /\left\langle R_{o}\right\rangle$ between 2.23 and 4.07 that will produce a scatter in Figure 11 only as the square root of this ratio, with the most extreme deviation from the linear function (gray line) being $20 \%$. In fact, $\left\langle R_{\mathrm{A}}\right\rangle /\left\langle R_{o}\right\rangle$ systematically changes, which explains the systematic scatter in Figure 11 as due to small differences in $\left\langle R_{\mathrm{A}}\right\rangle /\left\langle R_{o}\right\rangle$ for each temperature. The general trend in Figure 12 is that $\left\langle R_{\mathrm{A}}\right\rangle /\left\langle R_{o}\right\rangle$ increases for increasing flow temperature (see the solid cyan, yellow, and red lines), though that is not the case for simulations with $c_{S} / v_{\text {esc }}=0.2219$, for which the data points exhibit a peculiar behavior. Lastly, $\left\langle R_{\mathrm{A}}\right\rangle /\left\langle R_{o}\right\rangle$ should exhibit a dependence on the geometry of the field, and in particular, the expected trend is that for increasing complexity in the field geometry, this ratio decreases (see Finley \& Matt 2017).

Equation (33) can be further expanded by substituting $\bar{V}_{R_{\mathrm{A}}}$ with the velocity law, given by Equation (28), which yields

$$
\frac{\left\langle R_{\mathrm{A}}\right\rangle}{R_{*}}=\left(\frac{K_{l}^{4}}{K_{q}} \Upsilon\right)^{1 /(4+q)} .
$$

Equation (36) explains the fitting constants of Equation (15) in terms of the other fitting constants and represents an analytic formula of the torque-averaged Alfvén radius for any value of the parameter $\Upsilon$, for any known wind acceleration profile (known values of $K_{q}$ and $q$ ), for a dipolar field geometry, for a star that is a slow rotator, and for $\gamma=1.05$.

Finally, Equation (36) can work as a proxy to extract predictions for the values of $K_{s}$ and $m_{s}$ (see Equation (15)) that determine the simple power laws in Figure 7. It is expected that $K_{s} \sim\left(K_{l}^{4} / K_{q}\right)^{1 /(4+q)}$ and $m_{s} \sim 1 /(4+q)$ (see, for example, the fourth column in Table 3 for the predicted values of $m_{s}$ ) for each flow temperature. Undoubtedly, the primary reason for the differences in the four different power laws in Figure 7 is related to the acceleration of the flow, which depends on the stellar coronal temperature.

\section{Summary and Conclusions}

Employing 2.5D, ideal MHD, axisymmetric numerical simulations, we provide the first systematic study on how the thermodynamic conditions (i.e., flow temperature for the current work) in the stellar coronae of cool stars can influence the loss of stellar angular momentum due to magnetized winds. Our parameter space considers polytropic flows modified with rotation and magnetic fields, includes 30 steady-state wind solutions (see Appendix D for colorscale plots of the complete simulation grid), and quantifies the braking torque for four different coronal temperatures over a wide range of magnetic field strengths and for slow rotators, dipolar fields, and a fixed polytropic index $(\gamma=1.05)$. The following points summarize the main conclusions in this work.

1. For a given value of wind magnetization, $\Upsilon$, (or a given value of $\Upsilon_{\text {open }}$ ), a hotter wind is faster, reaches the Alfvén speed closer to the star and, as a consequence, the torque exerted on the surface of the star decreases.

2. We present two formulae that estimate the size of the torque-averaged Alfvén radius: one that depends on the parameter $\Upsilon$, which is based on stellar-surface parameters, and a second one that depends on $\Upsilon_{\text {open }}$, which is based on the amount of open magnetic flux. Each formulation gives a simple power law for each coronal temperature. By substituting Equation (15) into Equation (2), the stellar angular-momentum-loss rate due to a magnetized wind is

$$
\tau_{w}=K_{s}^{2} \Omega_{*} v_{\mathrm{esc}}^{-2 m_{s}} \dot{M}_{w}^{1-2 m_{s}} R_{*}^{2+4 m_{s}} B_{*}^{4 m_{s}}
$$

which is useful if the dipole field strength at the stellar surface is known. Similarly, by combining Equations (2) and (18), we have

$$
\tau_{w}=K_{o}^{2} \Omega_{*} v_{\mathrm{esc}}^{-2 m_{o}} \dot{M}_{w}^{1-2 m_{o}} R_{*}^{2-4 m_{o}} \Phi_{\text {open }}^{4 m_{o}},
$$

which is useful if the amount of the total open magnetic flux is known. The above relations can be used for studies of the rotational evolution of cool stars, and to predict the torque on stars with dipolar magnetic fields that are slow rotators and exhibit coronal winds with $\gamma=1.05$. Four different flow temperatures were studied, and the values of the fitting constants $K_{s}, m_{s}, K_{o}, m_{o}$ for each temperature can be found in Table 3 .

3. Using a simplified analysis (in Section 5), we identified that the wind acceleration profile is a key factor that determines how the torque scales with the parameter $\Upsilon$ or $\Upsilon_{\text {open. }}$. We found (in Figures 9 and 11) that by including the dimensionless velocity term $v_{\text {esc }} / \bar{V}_{R_{\mathrm{A}}}\left(\bar{V}_{R_{\mathrm{A}}}\right.$ is the wind's mean speed at the Alfvén surface) in each of the two torque formulae, all of the simulation data collapse into a unique power law, independent of the flow temperature. In other words, we propose that a key term that needs to be included in stellar torque prescriptions when one considers stars with different coronal conditions (and consequently different wind acceleration profiles) is the average wind speed at the Alfvén surface, regardless of how the outflow is heated and expands. This conclusion should be independent of the actual wind temperature or details of how the wind is driven, since the angular-momentum flux primarily depends on the flow velocity, mass density, and the magnetic field properties (see, e.g., Equations (13) and (14)).

4. By considering a power-law dependence of $\bar{V}_{R_{\mathrm{A}}}$ (i.e., the wind's mean speed at the Alfvén surface) in $\left\langle R_{\mathrm{A}}\right\rangle / R_{*}$, the torque-averaged Alfvén radius can be expressed with an analytic form (see Equations (31) and (36)), for a wellapproximated (or known) wind acceleration profile. 
Equations (2), (36), and (2), (31) then yield, respectively,

$$
\begin{aligned}
\tau_{w}= & \frac{K_{l}^{8 /(4+q)}}{K_{q}^{2 /(4+q)}} \Omega_{*} v_{\mathrm{esc}}^{-2 /(4+q)} \dot{M}_{w}^{(2+q) /(4+q)} \\
& \times R_{*}^{(12+2 q) /(4+q)} B_{*}^{4 /(4+q)},
\end{aligned}
$$

and

$$
\begin{aligned}
\tau_{w}= & \frac{K_{c}^{4 /(2+q)}}{K_{q}^{2 /(2+q)}} \Omega_{*} v_{\mathrm{esc}}^{-2 /(2+q)} \dot{M}_{w}^{q /(2+q)} \\
& \times R_{*}^{2 q /(2+q)} \Phi_{\mathrm{open}}^{4 /(2+q)} .
\end{aligned}
$$

These equations are successors to Equations (37) and (38) since they drop the dependence of magnetic braking on the flow temperature. Thus, Equations (39) and (40) should predict stellar torques for any given coronal temperature, but require the wind acceleration profile to be known. The values of the fitting constants $K_{q}, q$, which determine the acceleration of the outflow, for the temperatures examined in this study can be found in Table 3 (see also Appendix $\mathrm{C}$ for predictions on the values of these fitting constants over a continuous range of temperatures), while the values of $K_{c}, K_{l}$ are given in Sections 5.1 and 5.3, respectively.

In order to give an example of how our formulation can be used, we apply it to the solar case. In general, the torque exerted on the Sun (or any star) is an integrated quantity, and its value depends on a sum over the local values of the angularmomentum flux (see Equation (13)). During the solar minimum, the solar wind comprises two components, a fast and a slow wind (see also Section 1). Our wind models do not produce a bimodal outflow, and thus, we expect that our estimated solar torque should lie somewhere in between the torques predicted by our fastest (i.e., with $c_{s} / v_{\mathrm{esc}}=0.4$ ) and one of our slower wind models (i.e., with $c_{s} / v_{\text {esc }}=0.25$ ). To calculate the solar-wind torque, we will use the open-flux formula, given by Equation (38), because the open magnetic flux is measured in the solar wind by in situ spacecraft. Furthermore, previous studies (Réville et al. 2015a; Finley \& Matt 2017) showed this formulation to be independent of the field geometry at the surface (see also Equation (24)). Smith \& Balogh $(2003,2008)$ show that the open flux at solar minimum is typically $\sim 7 \times 10^{22} \mathrm{Mx}$. In addition, by using $\Omega_{\odot}=2.87 \times 10^{-6} \mathrm{rad} \mathrm{s}^{-1}$ and $\dot{M}_{\odot}=2 \times 10^{-14} M_{\odot} \mathrm{yr}^{-1}$, and the corresponding values of $K_{o}, m_{o}$ for $c_{s} / v_{\text {esc }}=0.25$ and 0.4 , Equation (38) yields angular-momentum-loss rates of $0.9 \times 10^{30}$ and $2.3 \times 10^{30} \mathrm{erg}$, respectively. These values agree with the solar braking rate found by Pizzo et al. (1983), which is $2.5-3.8 \times 10^{30} \mathrm{erg}$, and that found by $\mathrm{Li}$ (1999), which is $2.1 \times 10^{30} \mathrm{erg}$.

Even though we used simplified wind modeling (i.e., polytropic), the proposed torque formalism should work for any cool star with a known wind acceleration, mass-loss rate, and magnetic properties. The physical mechanisms that expand flows from the hot coronae of cool stars are still unknown (e.g., Cranmer 2012; Cranmer et al. 2015), but it is certain from early studies (e.g., Holzer 1977) that the physics of coronal heating is more complex than simple thermal-pressure expansion. The most modern ideas include Alfvén wave dissipation (e.g., Suzuki \& Inutsuka 2005; Cranmer et al. 2007; Sokolov et al. 2013; van der Holst et al. 2014), which work as an energy source and drive magnetized outflows. However, our full parameter space, with the range in flow temperatures that has been studied, should produce wind acceleration profiles within the range that exist in real stars.

Future work is needed to test the effects of more realistic wind physics (e.g., with variations in the polytropic index $\gamma$ or improved coronal heating models), and extending the study into the fast magnetic-rotator regime.

The authors thank Claudio Zanni, Victor Réville, Sasha Brun, Victor See, Adam Finley, and Matthew Gent for helpful discussions and comments on the manuscript. G.P. acknowledges support from the University of Exeter CEMPS through a $\mathrm{PhD}$ studentship. This project has received funding from the European Research Council (ERC) under the European Unions Horizon 2020 research and innovation programme (grant AWESoMeStars, agreement No. 682393). The authors also thank Andrea Mignone and others for the development and maintenance of the PLUTO code. This research has made use of NASA's Astrophysics Data System. All figures in this work were produced using the Python library Matplotlib (Hunter 2007).

\section{Appendix A Periodic Wind Solutions}

Each simulation is stopped when the solution relaxes to a steady state. About half of our wind solutions show a steady nature to some tolerance (see below), and the rest are periodic (or in a quasi-steady state) due to magnetic reconnections (due to numerical diffusion) at the neutral point (or cusp) located at the equatorial region of each simulation. As a consequence, a perfect steady-state solution cannot be obtained. Similar features have been noted by Washimi \& Shibata (1993), who found that the neutral point can have a non-steady behavior.

Due to this non-stationary nature of the equatorial region in some of our simulations, the fluxes passing through spherical surfaces, within our computational domain, are not constant in radius and time. As a result, the parameters $\mathrm{Y}$ and $\mathrm{Y}_{\mathrm{open}}$, and the effective Alfvén radius, $\left\langle R_{\mathrm{A}}\right\rangle / R_{*}$, show a dependence in both radius and time (whereas they should be constant for an ideal and steady-state MHD wind). However, the fluctuations of $\dot{M}_{w}, \tau_{w}$, and $\Phi_{\text {open }}$ are well-behaved and oscillatory, and the amplitude of the oscillations is constant in both $r$ and $t$. In order to derive single values for $\dot{M}_{w}, \tau_{w}$, and $\Phi_{\text {open, }}$, we used their median values in both $r$ (as discussed in Section 4.1) and $t$, where the value of a quantity was taken to be its median value after the initial transient phase of the simulation (i.e., typically after $\sim 10$ crossing times). These global values of $\dot{M}_{w}, \tau_{w}$ and $\Phi_{\text {open }}$ are then used to calculate $\Upsilon, \Upsilon_{\text {open }}$, and $\left\langle R_{\mathrm{A}}\right\rangle R_{*}$ for each case.

The errors of the time-varying $\Upsilon(t)$ and $\Upsilon_{\text {open }}(t)$ relative to the global values of $\Upsilon$ and $\Upsilon_{\text {open }}$ are shown in Figure 13 as a function of the number of wind crossing times, $t / t_{\text {cross }}$ (where $t_{\text {cross }}=50 R_{*} / v_{\text {esc }}$ ). The error of a given quantity relative to its median value is taken to be $\left(Q-Q_{\text {median }}\right) / Q_{\text {median }}$, where $Q$ is $\Upsilon$ or $\Upsilon_{\text {open. }}$. Two cases are presented (case 15 (23) has the magenta (blue) line). The solid lines correspond to the relative errors in $\Upsilon(t)$, and the dotted-dashed lines show the relative errors in $\Upsilon_{\text {open }}(t)$. From Figure 13, it is clear that $\Upsilon(t)$ and $\Upsilon_{\text {open }}(t)$ fluctuate in time, and furthermore are well-behaved functions of $t$. The variations in $\left\langle R_{\mathrm{A}}\right\rangle / R_{*}$ are smaller in 


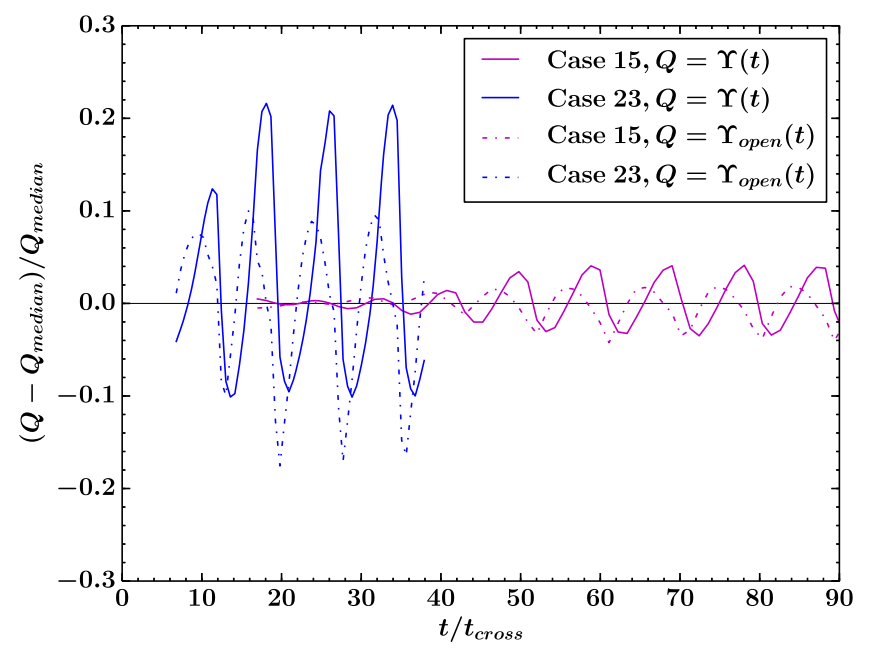

Figure 13. Variations of $\Upsilon(t)$ and $\Upsilon_{\text {open }}(t)$ relative to the median values of $\Upsilon$ and $\Upsilon_{\text {open }}$, respectively, vs. number of crossing times $t / t_{\text {cross. }}$. Two cases are shown, represented by the magenta lines (case 15) and the blue lines (case 23). The solid lines show the variations in parameter $\Upsilon$ and the dotted-dashed lines show the variations $\Upsilon_{\text {open }}$, respectively.

magnitude compared to the variations seen in $\Upsilon$ and $\Upsilon_{\text {open }}$ for a given wind solution. For example, case 23, shown in Figure 13, exhibit variations in $\left\langle R_{\mathrm{A}}\right\rangle / R_{*}$ of about $2 \%$ (compared to the range of variations in $\Upsilon$ shown in the figure).

Overall, for this study of 30 wind solutions, we obtained 16 steady-state wind solutions, meaning that the fluctuations in quantity $\Upsilon$, are not noticeable or less than $2 \%$. 7 wind solutions show variations in the range between $2 \%$ and $10 \%$, and in 7 simulations the variations in $\Upsilon(t)$ are between $10 \%$ and $30 \%$. Additionally, we did not see any systematic difference in the trends shown in this paper between the steady and periodic cases.

\section{Appendix B \\ Accuracy of the Numerical Solutions}

For ideal, axisymmetric, and steady-state MHD outflows, there are five scalar quantities (i.e., the derivative of the stream function or mass flux per magnetic flux, Bernoulli or energy function, entropy, specific angular momentum on a given stream function, and effective rotation rate of the field lines) that are constants of motion along each field line (e.g., Heinemann \& Olbert 1978; Lovelace et al. 1986; Ustyugova et al. 1999; Keppens \& Goedbloed 2000). In order to examine the accuracy of each of our numerical solutions, we check that each of the above quantities are conserved within some tolerance. As shown by Zanni \& Ferreira (2009), a difficult quantity to conserve and critical in order to measure accurate stellar torques is the effective rotation rate of the field lines, $\Omega_{\text {eff }}$. Solving Equation (9) for $\Omega_{*}$, the effective rotation of the field lines is defined as

$$
\Omega_{\mathrm{eff}}(\Psi) \equiv \frac{1}{r \sin \theta}\left(v_{\phi}-\frac{v_{p}}{B_{p}} B_{\phi}\right)
$$

where $\Psi$ is the magnetic stream function, given in spherical coordinates as $\Psi=r \sin \theta A$, where $r$ is the spherical radius and $A$ is the scalar magnetic field potential (i.e., $\boldsymbol{B}_{p}=\nabla \times A \hat{\phi}$ ).
Each field line has a unique value of $\Psi$. Since the stream function is a function of a scalar potential, $\Psi$ can be determined everywhere by specifying its value at a single point. We choose $\Psi$ to be zero at the pole on the stellar surface (i.e., $\Psi=0$ for $\theta=0$ and $r=R_{*}$ ), and as a result, the first polar field line will have a $\Psi$ value of zero.

In the ideal MHD regime, for any axisymmetric and steadystate wind solution, Equation (9) should hold throughout the numerical domain, and the plasma, which flows along the field lines, should rotate such that the ratio $\Omega_{\text {eff }} / \Omega_{*}$ is equal to unity. Any deviations from this value occur due to numerical diffusion and non-stationary wind solutions. The crucial ingredients to achieve the correct rotation for the matter around the star are the boundary conditions on $v_{\phi}$ and $B_{\phi}$, imposed on the inner boundary (i.e., stellar surface) of the computational domain, as pointed out in Zanni \& Ferreira (2009). For our simulations, the toroidal speed of the plasma is enforced in the stellar boundary via Equation (9) and $B_{\phi}$ is linearly extrapolated (i.e., $\partial B_{\phi} / \partial r=$ const.) into the ghost zones, a boundary condition that works well for the current stellar-wind numerical setup (for a more detailed discussion on different boundary conditions on $B_{\phi}$, see also Zanni \& Ferreira 2009).

In Figure 14, the behavior of the normalized effective rotation rate is presented as a $2 \mathrm{D}$ colorscale plot (top panels) and in a $\Omega_{\text {eff }} / \Omega_{*}$ versus $\Psi$ plot (bottom panels) for two numerical wind solutions of the study. The two cases shown are one that is typical (case 6) and one (case 9) that exhibits among the largest errors in the conservation of $\Omega_{\mathrm{eff}}$. In the top panels of Figure 14, the regions in the plots colored with gray correspond to an $\Omega_{\text {eff }} / \Omega_{*}$ that is equal to unity. The blue and red regions correspond to $\Omega_{\mathrm{eff}} / \Omega_{*}<1$ and $\Omega_{\mathrm{eff}} / \Omega_{*}>1$, respectively. For example, in case 6 , we identify that $\Omega_{\mathrm{eff}} / \Omega_{*}$ is not conserved along field lines located at midlatitudes, adjacent to the dead zone, where steep gradients of $v_{\text {pol }}$ and $B_{\phi}$ enhance the numerical diffusion. A measure of how $\Omega_{\mathrm{eff}} / \Omega_{*}$ deviates from unity, for these two simulations, is given in the bottom panels of Figure 14. Each point in the bottom panels represents a grid cell, within our domain, and every value of $\Psi$ corresponds to a different field line. Values of $\Psi$ from 0 to about 0.1 (case 6), and from 0 to about 0.25 (case 9) correspond to open-field lines, in which the wind flows outwards, and the rest of the $\Psi$ values represent closed magnetic loops. For case 6 , we observe that some open-field lines subrotate (up to $40 \%$ ) and some closed-field lines overrotate (up to $30 \%$ ). A comparison of $\Omega_{\mathrm{eff}} / \Omega_{*}$ between the two cases reveals that the errors for case 9 (and cases with a high wind magnetization) are shifted to the left because such simulations produce less fractional open flux. For these cases, the dead zones are more extended and cover most of the stellar surface, and as a result most of the open-field lines are influenced by numerical errors. This can be easily seen in the top-right panel in which the grayshaded regions decrease significantly compared to typical cases with median or low values of $\Upsilon$ (top-left panel). Furthermore, the amplitude of the errors becomes larger in case 9 (see the bottom-left and right panels) as a consequence of a wind that is more magnetized, and due to this, faster (i.e., with even steeper gradients of $v_{\text {pol }}$ and $B_{\phi}$ ). In other words, numerical errors are more significant in simulations with high wind magnetization.

One way to reduce these non-ideal features is to increase the resolution of the computational domain. For example, our resolution studies (not shown) indicate that by doubling the 

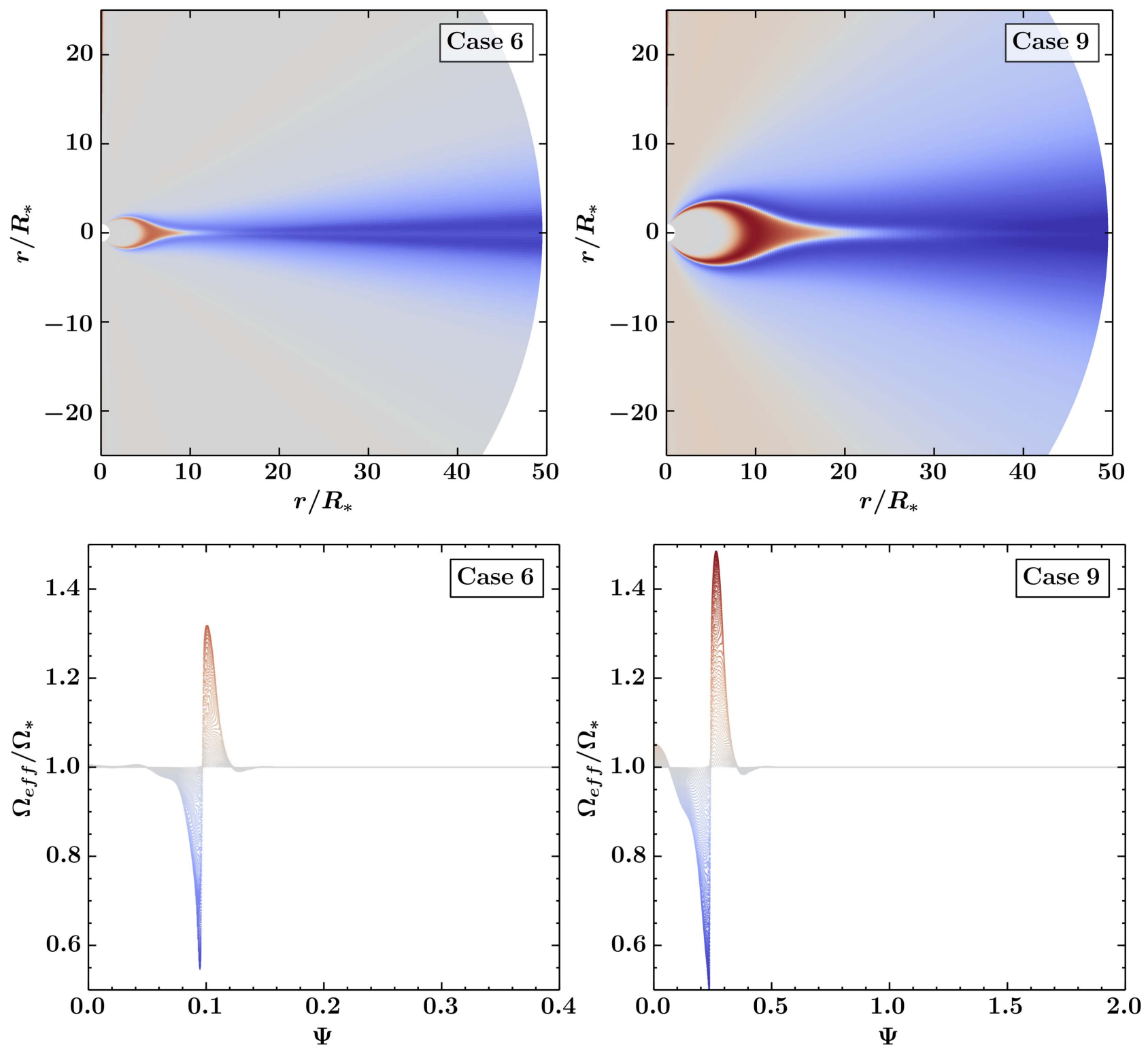

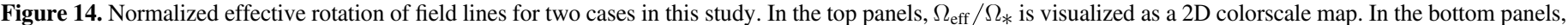

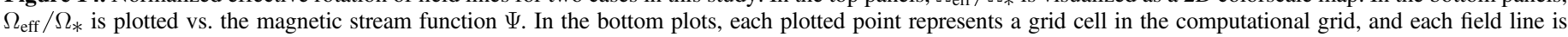

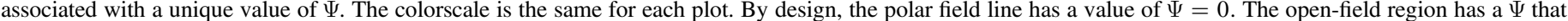
varies between 0 and 0.1 for case 6 (bottom-left panel), and between 0 and 0.25 for case 9 (bottom-right panel).

number of cells in the $\theta$ direction, numerical errors in $\Omega_{\text {eff }} / \Omega_{*}$ decrease, but the torque-averaged $\left\langle R_{\mathrm{A}}\right\rangle / R_{*}$ for most cases increase only by a few percent. Bigger differences in the values of $\left\langle R_{\mathrm{A}}\right\rangle / R_{*}$, due to a higher grid resolution, are observed in simulations with $\Upsilon$ above $10^{4}$, but even for these cases, $\left\langle R_{\mathrm{A}}\right\rangle / R_{*}$ does not increase by more than $10 \%$. These systematic errors suggest that a more accurate numerical treatment would lead to slightly steeper power laws in the trends shown in Figures 7-9 and 11.

\section{Appendix C}

\section{Toward Predicting Torque for Any Temperature}

In this appendix, we present empirical relations that predict the fitting constants $K_{q}, q$ used to prescribe the wind speed at the Alfvén radius (see Equation (28)) as functions of the input parameter $c_{s} / v_{\text {esc }} . K_{q}$ and $q$ are needed in order to estimate the torque-averaged Alfvén radius (see Equations (31) and (36)), and since our study investigated only four different flow temperatures and their corresponding wind acceleration profiles, our aim is to provide a practical method that could give $K_{q}$ and $q$ over a larger and continuous range of $c_{s} / v_{\text {esc }}$. This method should work for any continuous range of $c_{s} / v_{\text {esc }}$ for polytropic winds with $\gamma=1.05$. It also suggests how to generalize for other winds, but we do not test that here.

The values of $K_{q}$ and $q$ versus the parameter $c_{s} / v_{\text {esc }}$ are shown in Figure 15 for our four temperatures (blue circles). All of the values of $q$ are positive in the range between zero and unity. There is no physical reason for not getting wind solutions with values of $q$, such as $q>1$, but $q=0$ is the lower limit for any accelerating flow. Regardless of the obvious 

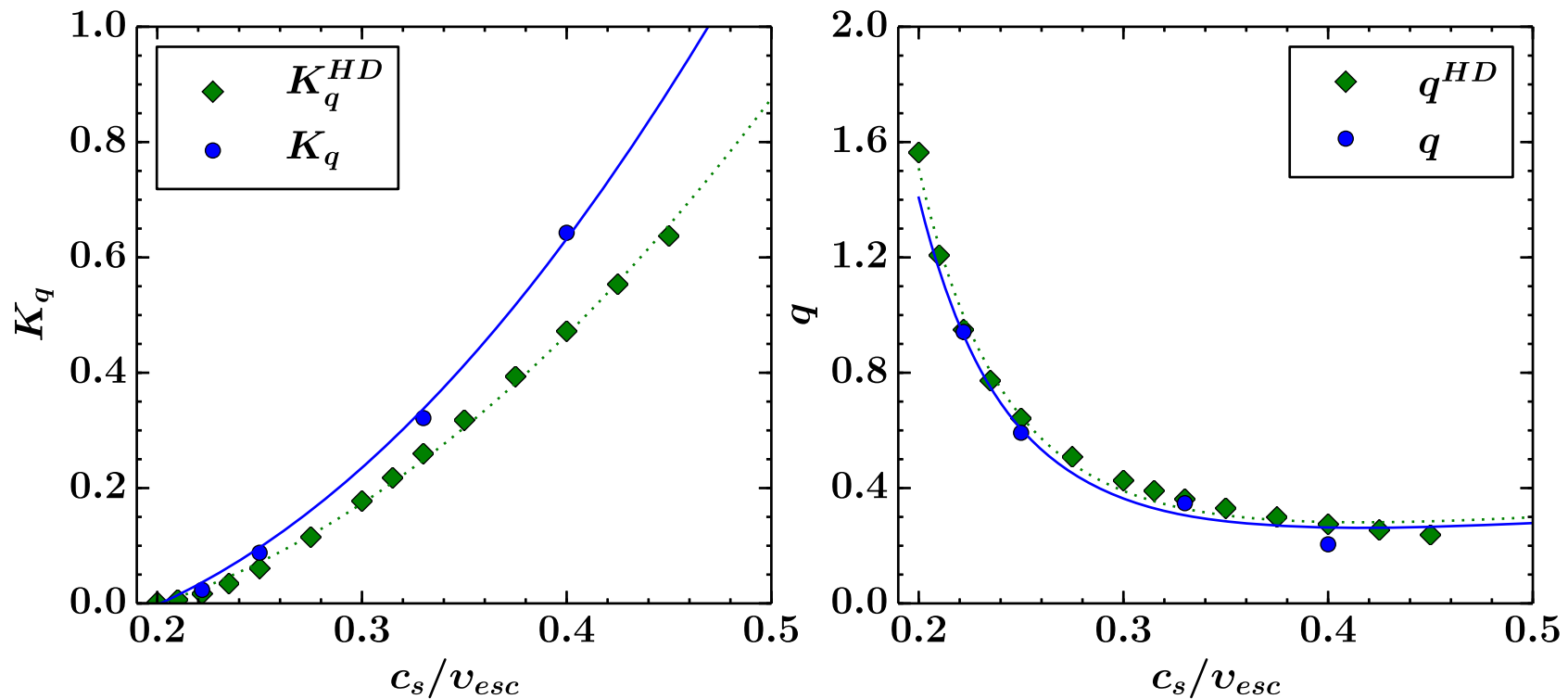

Figure 15. Fitting constants $K_{q}$ (left panel) and $q$ (right panel) of Equation (28) vs. the parameter $c_{s} / v_{\text {esc }}$. The blue circles correspond to the values of $K_{q}$ and $q$ from the velocity laws presented in Figure 10. The green diamonds correspond to the fitting constants $K_{q}^{\mathrm{HD}}$ and $q$, which have been obtained from $1 \mathrm{D}$ hydrodynamic wind speed profiles. The dotted lines fit the green data points, according to Equations (42) and (43). The blue solid lines show the fitting functions (Equations (44) and (45)) for $K_{q}$ and $q$, respectively.

trends in Figure 15 (i.e., $K_{q}$ and $q$ monotonically increase and decrease with increasing $c_{s} / v_{\text {esc }}$, respectively), any function that could possibly represent (or fit) these data points would be rather biased due to the small number of data points (only four). Therefore, in order to construct functions that can fit the data in Figure 15, we employ the following approach. In Figure 10, we demonstrated that the $\bar{V}_{R_{\mathrm{A}}} / v_{\text {esc }}$ versus $\left\langle R_{\mathrm{A}}\right\rangle / R_{*}$ data points exhibit a behavior similar to the shape of the radial velocity profiles (i.e., $v_{r} / v_{\text {esc }}$ versus $r / R_{*}$ ) of the $1 \mathrm{D}$ hydrodynamic winds shown in Figures 1 and 10. Based on that, one can infer what $\bar{V}_{R_{\mathrm{A}}}$ would be for any given flow temperature (or any given value of $c_{S} / v_{\text {esc }}$ ) from polytropic Parker's winds with that value of $c_{s} / v_{\text {esc }}$. Hence, we produce 14 Parker's wind models, in which the parameter $c_{s} / v_{\text {esc }}$ varies between 0.2 and 0.45 . The velocity profiles of these winds are functions of radial distance from the surface of the star. Then, we treat any radial distance of these profiles as a potential Alfvén radius and its corresponding flow velocity as the mean speed of the outflow at the Alfvén radius (i.e., $\bar{V}_{R_{\mathrm{A}}}$ ). Following Equation (28), we fit these hydrodynamic wind speed profiles, assuming that the flow speed is a power law in radial distance (i.e., $\left.v(r) \propto K_{q}^{\mathrm{HD}} r^{q^{\mathrm{HD}}}\right)$. Since for the entire study the minimum and maximum values of $\left\langle R_{\mathrm{A}}\right\rangle / R_{*}$ are $3.27 R_{*}$ and $25.3 R_{*}$, respectively, the hydrodynamic wind speed profiles are fitted for radial distances that range between $4 R_{*}$ and $25 R_{*}$. We obtain 14 new pairs of the dimensionless fitting constants $K_{q}^{\mathrm{HD}}$ and $q^{\mathrm{HD}}$, also shown in Figure 15 as green diamonds. The values of $K_{q}^{\mathrm{HD}}$ and $q^{\mathrm{HD}}$ can be slightly influenced by considering a different range in radii in order to fit these hydrodynamic wind speed profiles. The following empirical functions can fit the new data points (i.e., $K_{q}^{\mathrm{HD}}$ and $q^{\mathrm{HD}}$ )

$$
\begin{gathered}
K_{q}^{\mathrm{HD}}=\alpha_{1}\left(c_{s} / v_{\mathrm{esc}}\right)^{2}+\beta_{1}\left(c_{s} / v_{\mathrm{esc}}\right), \\
q^{\mathrm{HD}}=\alpha_{2}\left(c_{s} / v_{\mathrm{esc}}\right)^{\nu_{2}}+\beta_{2}\left(c_{s} / v_{\mathrm{esc}}\right),
\end{gathered}
$$

where $\alpha_{1}, \alpha_{2}, \beta_{1}, \beta_{2}$, and $\nu_{2}$ are fitting coefficients. The bestfit values are $\alpha_{1}=5.87$ and $\beta_{1}=-1.18$ for Equation (42), and $\alpha_{2}=0.000979, \nu_{2}=-4.51$, and $\beta_{2}=0.553$ for Equation (43).
Equations (42) and (43) are represented in both panels of Figure 15 by the green dotted curves.

Equations (42) and (43) can represent the four data points (blue circles) in Figure 15 just by including a multiplicity factor. Indeed, the blue solid lines in Figure 15 show that the data of $K_{q}$ and $q$ can be fitted by functions in the form of

$$
\begin{aligned}
& K_{q}=D_{1}\left[\alpha_{1}\left(c_{s} / v_{\mathrm{esc}}\right)^{2}+\beta_{1}\left(c_{s} / v_{\mathrm{esc}}\right)\right] \\
& q=D_{2}\left[\alpha_{2}\left(c_{s} / v_{\mathrm{esc}}\right)^{\nu_{2}}+\beta_{2}\left(c_{s} / v_{\mathrm{esc}}\right)\right]
\end{aligned}
$$

where again $D_{1}, D_{2}$ are fitting constants and their best-fit values are found to be $D_{1}=1.36$ and $D_{2}=0.932$. In conclusion, Equations (44) and (45) can successfully predict the values of the dimensionless fitting constants $K_{q}$ and $q$ for any value of the parameter $c_{s} / v_{\text {esc }}$ in the range between 0.2 and 0.45 for thermally driven winds from slow-rotating stars, with dipolar fields, and a fixed value of the polytropic index equal to 1.05.

\section{Appendix D Complete Grid of Simulations}

Figure 16 presents colorscale plots of the wind's poloidal velocity for all numerical solutions in this study. Cases 1 to 9,10 to 16,17 to 23 , and 24 to 30 have, respectively, $c_{s} / v_{\mathrm{esc}}=0.2219,0.25,0.33$, and 0.4 . Each panel in Figure 16 shows the full computational grid, the location, and the shape of the wind's critical surfaces. The sonic and Alfvén surfaces are depicted with white and red solid lines, respectively. The magenta dashed lines show the effective lever arm, $\left\langle R_{\mathrm{A}}\right\rangle / R_{*}$, that brakes the stellar rotation. A different coronal temperature (primarily) and a higher wind magnetization (to a lesser extent) affects the outflow speed and acceleration profile. This feature can be seen in the changes in the colorscale of each panel. Overall, for a given value of the wind magnetization, $\Upsilon$, a hotter wind reaches the Alfvén surface in a shorter distance from the stellar surface, the size of the lever arm decreases, and as a result, the magnetic torque is reduced. 


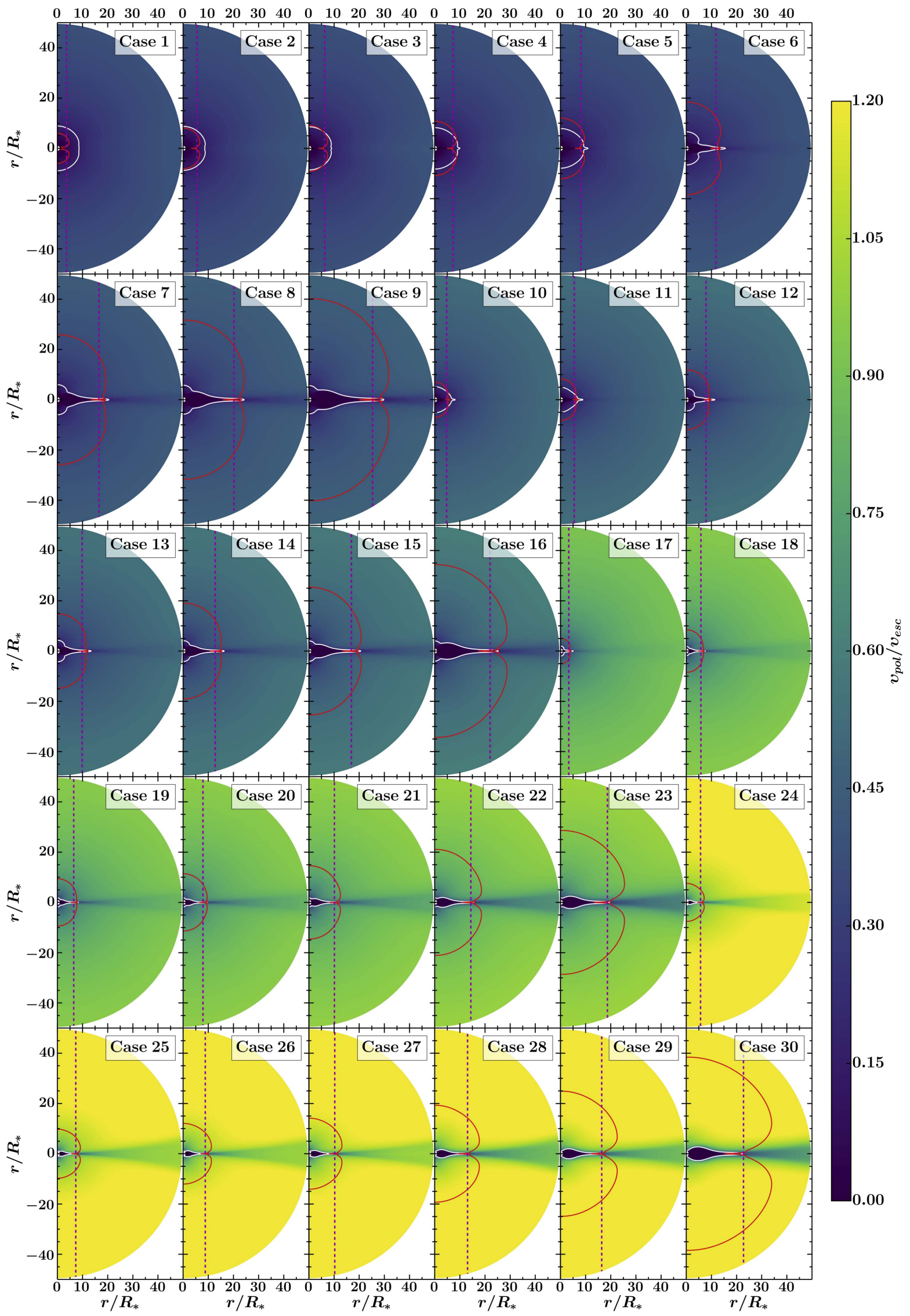

Figure 16. Wind poloidal velocity color maps of the entire study. The white and red lines represent the sonic and Alfvén surfaces, respectively. The magenta dashed lines show the location of the torque-averaged Alfvén radius (or effective lever arm). Simulations 1 to 9,10 to 16,17 to 23 , and 24 to 30 have, respectively, $c_{s} / v_{\mathrm{esc}}=0.2219,0.25,0.33$, and 0.4 . 


\section{ORCID iDs}

George Pantolmos (10 https://orcid.org/0000-0001-7788-3727

Sean P. Matt (1) https://orcid.org/0000-0001-9590-2274

\section{References}

Alvarado-Gómez, J. D., Hussain, G. A. J., Cohen, O., et al. 2016, A\&A, 594, A95

Amard, L., Palacios, A., Charbonnel, C., Gallet, F., \& Bouvier, J. 2016, A\&A, 587, A105

Aschwanden, M. J. 2005, Physics of the Solar Corona. An Introduction with Problems and Solutions (2nd ed.; Berlin: Springer)

Balsara, D. S., \& Spicer, D. S. 1999, JCoPh, 149, 270

Baraffe, I., Chabrier, G., Allard, F., \& Hauschildt, P. H. 1998, A\&A, 337, 403

Barnes, S. A. 2003, ApJ, 586, 464

Barnes, S. A. 2010, ApJ, 722, 222

Belcher, J. W., \& MacGregor, K. B. 1976, ApJ, 210, 498

Bouvier, J., Matt, S. P., Mohanty, S., et al. 2014, in Protostars and Planets VI, ed. H. Beuther et al. (Tucson, AZ: Univ. Arizona Press), 433

Cohen, O., \& Drake, J. J. 2014, ApJ, 783, 55

Cohen, O., Sokolov, I. V., Roussev, I. I., et al. 2007, ApJL, 654, L163

Cranmer, S. R. 2012, SSRv, 172, 145

Cranmer, S. R., Asgari-Targhi, M., Miralles, M. P., et al. 2015, RSPTA, 373, 20140148

Cranmer, S. R., \& Saar, S. H. 2011, ApJ, 741, 54

Cranmer, S. R., van Ballegooijen, A. A., \& Edgar, R. J. 2007, ApJS, 171, 520

De Moortel, I., \& Browning, P. 2015, RSPTA, 373, 20140269

Donati, J.-F., \& Brown, S. F. 1997, A\&A, 326, 1135

Donati, J.-F., \& Landstreet, J. D. 2009, ARA\&A, 47, 333

Finley, A. J., \& Matt, S. P. 2017, ApJ, 845, 46

Fisk, L. A. 2003, JGRA, 108, 1157

Gallet, F., \& Bouvier, J. 2013, A\&A, 556, A36

Gallet, F., \& Bouvier, J. 2015, A\&A, 577, A98

Garraffo, C., Drake, J. J., \& Cohen, O. 2015, ApJL, 807, L6

Garraffo, C., Drake, J. J., \& Cohen, O. 2016, A\&A, 595, A110

Hansteen, V. H., \& Velli, M. 2012, SSRv, 172, 89

Heinemann, M., \& Olbert, S. 1978, JGR, 83, 2457

Holzer, T. E. 1977, JGR, 82, 23

Holzwarth, V., \& Jardine, M. 2007, A\&A, 463, 11

Hunter, J. D. 2007, CSE, 9, 90

Irwin, J., \& Bouvier, J. 2009, in IAU Symp. 258, The Ages of Stars, ed. E. E. Mamajek, D. R. Soderblom, \& R. F. G. Wyse (Cambridge: Cambridge Univ. Press), 363

Johnstone, C. P., Güdel, M., Brott, I., \& Lüftinger, T. 2015a, A\&A, 577, A28

Johnstone, C. P., Güdel, M., Lüftinger, T., Toth, G., \& Brott, I. 2015b, A\&A, 577, A27

Kawaler, S. D. 1988, ApJ, 333, 236

Keppens, R., \& Goedbloed, J. P. 1999, A\&A, 343, 251

Keppens, R., \& Goedbloed, J. P. 2000, ApJ, 530, 1036

Klimchuk, J. A. 2015, RSPTA, 373, 20140256

Kopp, R. A., \& Holzer, T. E. 1976, SoPh, 49, 43

Kraft, R. P. 1967, ApJ, 150, 551

Lamers, H. J. G. L. M., \& Cassinelli, J. P. 1999, in Introduction to Stellar Winds (Cambridge: Cambridge Univ. Press), 452

Leer, E., \& Holzer, T. E. 1980, JGR, 85, 4681

Li, J. 1999, MNRAS, 302, 203

Lovelace, R. V. E., Mehanian, C., Mobarry, C. M., \& Sulkanen, M. E. 1986, ApJS, 62, 1

Low, B. C., \& Tsinganos, K. 1986, ApJ, 302, 163

Lüftinger, T., Vidotto, A. A., \& Johnstone, C. P. 2015, in Astrophysics and Space Science Library 411, Characterizing Stellar and Exoplanetary Environments, ed. H. Lammer \& M. Khodachenko (Berlin: Springer), 37

Matt, S., \& Pudritz, R. E. 2008, ApJ, 678, 1109

Matt, S. P., Brun, A. S., Baraffe, I., Bouvier, J., \& Chabrier, G. 2015, ApJL, 799, L23

Matt, S. P., MacGregor, K. B., Pinsonneault, M. H., \& Greene, T. P. 2012, ApJL, 754, L26

McComas, D. J., Ebert, R. W., Elliott, H. A., et al. 2008, GeoRL, 35, L18103

McComas, D. J., Elliott, H. A., Schwadron, N. A., et al. 2003, GeoRL, 30,1517

McComas, D. J., Velli, M., Lewis, W. S., et al. 2007, RvGeo, 45, RG1004

Meibom, S., Barnes, S. A., Latham, D. W., et al. 2011, ApJL, 733, L9
Meibom, S., Barnes, S. A., Platais, I., et al. 2015, Natur, 517, 589

Mestel, L. 1968, MNRAS, 138, 359

Mestel, L. 1984, in Cool Stars, Stellar Systems, and the Sun, ed. S. L. Baliunas \& L. Hartmann (Berlin: Springer), 49

Mestel, L. 1999, Stellar Magnetism (New York: Oxford Univ. Press)

Mestel, L., \& Spruit, H. C. 1987, MNRAS, 226, 57

Mignone, A., Bodo, G., Massaglia, S., et al. 2007, ApJS, 170, 228

Mikić, Z., Linker, J. A., Schnack, D. D., Lionello, R., \& Tarditi, A. 1999, PhPl, 6, 2217

Ofman, L. 2004, AdSpR, 33, 681

Ofman, L. 2010, LRSP, 7, 4

Owocki, S. 2009, in EAS Publications Ser. 39, Stellar Magnetism, ed C. Neiner \& J.-P. Zahn (Trieste: EDP Sciences), 223

Parker, E. N. 1958, ApJ, 128, 664

Parker, E. N. 1963, Interplanetary Dynamical Processes (New York: Interscience)

Pizzo, V., Schwenn, R., Marsch, E., et al. 1983, ApJ, 271, 335

Pizzolato, N., Maggio, A., Micela, G., Sciortino, S., \& Ventura, P. 2003, A\&A, 397,147

Pneuman, G. W. 1966, ApJ, 145, 242

Pneuman, G. W., \& Kopp, R. A. 1971, SoPh, 18, 258

Powell, K. G., Roe, P. L., Linde, T. J., Gombosi, T. I., \& Zeeuw, D. L. D. 1999, JCoPh, 154, 284

Priest, E. 2014, Magnetohydrodynamics of the Sun (Cambridge: Cambridge Univ. Press)

Reiners, A., \& Mohanty, S. 2012, ApJ, 746, 43

Réville, V., Brun, A. S., Matt, S. P., Strugarek, A., \& Pinto, R. F. 2015a, ApJ, 798, 116

Réville, V., Brun, A. S., Strugarek, A., et al. 2015b, ApJ, 814, 99

Réville, V., Folsom, C. P., Strugarek, A., \& Brun, A. S. 2016a, ApJ, 832, 145

Réville, V., Folsom, C. P., Strugarek, A., \& Brun, A. S. 2016b, in 19th Cambridge Workshop on Cool Stars, Stellar Systems, and the Sun, ed. G. A. Feiden (Genève: CERN), 33

Riley, P., Linker, J. A., Mikić, Z., et al. 2006, ApJ, 653, 1510

Sakurai, T. 1985, A\&A, 152, 121

Schatzman, E. 1962, AnAp, 25, 18

Schwadron, N. A., \& McComas, D. J. 2003, ApJ, 599, 1395

Schwadron, N. A., \& McComas, D. J. 2008, ApJL, 686, L33

See, V., Jardine, M., Vidotto, A. A., et al. 2015, MNRAS, 453, 4301

See, V., Jardine, M., Vidotto, A. A., et al. 2017a, MNRAS, 466, 1542

See, V., Jardine, M., Vidotto, A. A., et al. 2017b, MNRAS, in press

Skumanich, A. 1972, ApJ, 171, 565

Smith, E. J., \& Balogh, A. 2003, in AIP Conf. Ser. 679, Solar Wind Ten, ed. M. Velli (Melville, NY: AIP), 67

Smith, E. J., \& Balogh, A. 2008, GeoRL, 35, L22103

Sokolov, I. V., van der Holst, B., Oran, R., et al. 2013, ApJ, 764, 23

Suzuki, T. K., Imada, S., Kataoka, R., et al. 2013, PASJ, 65, 98

Suzuki, T. K., \& Inutsuka, S.-i. 2005, ApJL, 632, L49

Testa, P., Saar, S. H., \& Drake, J. J. 2015, RSPTA, 373, 20140259

Toro, E. F. 2009, Riemann Solvers and Numerical Methods for Fluid Dynamics (Berlin: Springer)

Tout, C. A., \& Pringle, J. E. 1992, MNRAS, 256, 269

ud-Doula, A., \& Owocki, S. P. 2002, ApJ, 576, 413

Ud-Doula, A., Owocki, S. P., \& Townsend, R. H. D. 2009, MNRAS, 392, 1022

Ustyugova, G. V., Koldoba, A. V., Romanova, M. M., Chechetkin, V. M., \& Lovelace, R. V. E. 1999, ApJ, 516, 221

van der Holst, B., Sokolov, I. V., Meng, X., et al. 2014, ApJ, 782, 81

Velli, M., Pucci, F., Rappazzo, F., \& Tenerani, A. 2015, RSPTA, 373, 20140262

Vidotto, A. A., Gregory, S. G., Jardine, M., et al. 2014a, MNRAS, 441, 2361

Vidotto, A. A., Jardine, M., Morin, J., et al. 2014b, MNRAS, 438, 1162

Vidotto, A. A., Opher, M., Jatenco-Pereira, V., \& Gombosi, T. I. 2009, ApJ, 699,441

Wang, Y.-M., \& Sheeley, N. R., Jr. 1991, ApJL, 372, L45

Washimi, H., \& Shibata, S. 1993, MNRAS, 262, 936

Weber, E. J., \& Davis, L., Jr. 1967, ApJ, 148, 217

Wood, B. E., Müller, H.-R., Redfield, S., \& Edelman, E. 2014, ApJL, 781 L33

Wood, B. E., Müller, H.-R., Zank, G. P., \& Linsky, J. L. 2002, ApJ, 574 412

Wright, N. J., Drake, J. J., Mamajek, E. E., \& Henry, G. W. 2011, ApJ, 743 48

Zanni, C., \& Ferreira, J. 2009, A\&A, 508, 1117 